\title{
The influence of certain astronomical and constructive parameters on the concentration of solar radiation with plane heliostats fields
}

\author{
V. Bădescu \\ Energetica, Centrale Electrice, Inst. Polytechnique de Bucharest, Bucharest 79590, Romania
}

(Reçu le 23 février 1984, révisé les 14 décembre 1984 et 29 janvier 1985, accepté le 25 mars 1985)

\begin{abstract}
Résumé. - Dans cet article nous étudions les influences des quelques paramètres astronomiques, et de construction sur le fonctionnement d'une centrale solaire à tour et champ d'héliostats. Les paramètres astronomiques sont : (1) l'heure et $(2)$ le jour. Les paramètres de construction sont : $(3,4)$ les distances entre héliostats dans les directions nord-sud et est-ouest, (5) la grandeur du miroir, (6) l'hauteur de la tour, (7) la distance entre la tour et le champ d'héliostats. Les aspects suivants du fonctionnement de la centrale solaire : (i) l'orientation des miroirs, (ii) les performances optiques des héliostats et (iii) la concentration de la radiation solaire ont été étudiés.
\end{abstract}

\begin{abstract}
In this paper, we present the influence of some astronomical and constructive parameters on the HFT working. The astronomical parameters are : (1) hour and (2) day. The constructive parameters are : $(3,4)$ the east-west and north-south distances between heliostats, (5) the mirror size, (6) the tower height, (7) the distance between the tower and the heliostats field. The HFT working is analysed from three points of view : (i) the mirrors orientation, (ii) the heliostats optical performances and (iii) the radiation concentration.
\end{abstract}

\section{Introduction.}

The concentration of solar radiation by means of heliostats fields with tower (HFT) has actually a great variety of constructive and functional technical solutions. This situation is due both, to the fact that the existing designs and achievements reach different goals and performances and, that the subject is still under consideration. The constructive solutions related to the geometrical part of a HFT are principally differentiated by the number of heliostats, which may vary from several tens [1] up to a couple of ten-thousand $[2,3]$, the mirrors form, which can be square [1, 3-8], rectangular [9], polygonal [10] or circular [5, 11], the mirror surface area, which varies between 4 and $50 \mathrm{~m}^{2}[1,3,4,7,9,19]$, and by the mirror surface curvature, which can be spherical, toroidal, paraboloidal $[5,8]$ or plane. The area of the heliostats fields varies between several thousands of square meters [5] up to several millions of square meters [2,3], while the field form is either rectangular or square $[2,3,9]$, circular [13] or sometimes more complicated [5, 14]. The tower on which the receiver is placed is located either in the south or in the centre of the heliostats field $[1,5,8,14,15]$, and its height is in the range of several thousand meters $[8,9]$ and $450 \mathrm{~m}[2,3]$. The receiver can be parallelipipedical [1, 14], spherical [3, 17], cylindrical [16] or have a more complicated form [18].
Among the functional solutions, which are not really considered in the present paper, we only mention those which are differentiated by the nature of the orientational systems (see for example $[9,12,19,20]$ ) and by the nature of the working fluid (see for example [13]).

The simple enumeration of the remark above, reveals that for the time being, there still are differences of principle on certain aspects of a HFT, fact which has been already noted by other authors [8, 17]. Further on, we do not intend to discuss these differences of principle, taking into account a specified HFT case.

The HFT model which will be studied here represents one of the variants analysed during the preliminary studies performed by us in order to project a solar power plant [21]. The heliostats field is entirely placed on a horizontal surface having a rectangular form, with one of its symmetry axis in the north-south direction. The mirrors are disposed at equal distances in parallel strings on the east-west and respectively north-south directions. On the prolongation of the north-south symmetry axis of the heliostats field is the tower on which the receiver is placed. This receiver is of a cavity type. The mirrors used are plane, with a square form. The system of the heliostat alignment has two rotation axes, to correct the angles of elevation and azimuth. 
The more important parameters influencing the process of radiation concentration using a HFT, are the astronomical parameters (prescribed) and the constructive parameters (given above). A HFT design supposes essentially the correct determination of the values of the constructive parameters so that, under specified conditions determined by the values of the astronomical parameters a certain target function can be reached (collected power as high as possible [15], a spatial and temporary uniformity of the concentrated flux [22], a covering of the field surface as efficient as possible [8]).

The two categories of parameters influence the following aspects of the HFT : (i) the heliostats orientation; (ii) the optical performances of the heliostats; (iii) the radiation concentration on the receiver surface. Among the aspects mentioned above, a special attention was given by most of the authors on the third point (iii), on the one hand because of its practical importance and on the other hand because of the complexity of the required computing technique. Thus, a series of computing programs of the radiation distribution on the receiver surface were developed, being fundamented on complete models of the analysed physical process (Lipps [6], McFee [3], Riaz and Gurr [16], Lipps and Walzel [10], Lipps [23], Walzel and Lipps [24]). Nevertheless, these computing programs are expensive, because of the corrective effects, and determine weakly convergent numerical procedures $[10,25]$. For example we quote the program FLASH which requires $240 \mathrm{~s}$ to compute only one image of the flux concentrated by a heliostats field formed of 120 mirrors [10]. Under these circumstances it is often advantageous to use simplified programs for computing the intensity of the concentrated radiation. So, to study the receiver interception factor, Lipps and Walzel [10] used the program HCOEF, because in spite of its lower precision, it is 116 times faster than the sophisticated program FLASH. Also, to analyse the most efficient of the heliostat movement, Igel and Hughes [5] use a simple technique, preferred to the complete computer program HELIOS, which is only used to solve accuracy problems. Simplified programs were also used by Le Phat Vinh [8], to compare the performances of plane and respectively curved heliostats, Wei [25] and Abatut and Achaibou [15] to study the global optical efficiency of the heliostats field, Bădescu and Vasiloiu [26] for the approach of the thermal shocks in the receiver. The computing technique from [26] is also used in this paper.

The present study intends to bring to evidence the influence of certain astronomical and constructive parameters on the process of solar radiation concentration by means of HFT. The following sections present the basis of the computing method on which the SOLA program is structured, as well as the simulation technique. Then, we show our results. During the discussions we may make reference to literature, if some particular cases studied by other authors can be compared to the model analysed by us. The references have a rather relative character because there is not a full correspondence between the parameters used by us and those used by the authors quoted in order to characterize the HFT. At the end of the paper, a few remarks concerning the precision of the results are made.

\section{The model of solar radiation concentration in HFT.}

\subsection{Preliminary NOtions AND Definitions. - We} name optical centre (focus, focal centre) a point placed at the cavity aperture, where we intend to obtain the maximum concentration of the radiation. We denominate by optical axis of a heliostat, the straight line joining the mirror centre $\mathrm{O}_{1}$ with the optical centre of the system. The main incident ray is the one joining the solar disk centre to the mirror centre. By reflection, a main incident ray becomes a main reflected ray. We consider that a heliostat is aligned (oriented) if the main incident ray reflects in the optical centre. By alignment (orientation) condition we understand the relation which must exist between the directions of the main incident ray, the normal to the mirror plane in the point $\mathrm{O}_{1}$ and respectively the main reflected ray, in order to align (orientate) the heliostat. We consider a heliostat as being plane or curved if its mirror has a plane, respectively curved surface.

Because the HFT receiver is of cavity type and the tower is placed in the south of the heliostats field, it is convenient to define an image (focal) plane [8]. The image plane is vertical, has the east-west direction and passes through the optical centre. The intersection of the radiation beam reflected by a mirror with the focal plane determines a polygonal figure named image. We consider an image to be ideal if it was determined neglecting the disturbing phenomena presented in $\S 2.2$. In case of mirrors with square surface, generally the image is a parallelogram. Superposition of the heliostats images determines in the focal plane, a geometrical figure named total image or the image of the heliostats field. We call equal concentration region (ECR) the domain of the image plane where all of the field heliostats reflect.

2.2 THE PHYSICAL MODEL AND THE MATHEMATICAL MODEL PRINCIPLES. - The model built by us to describe the heliostats field belongs to the category of discontinuous models (see for example $[3,5,6,10]$ ), which estimate separately the contribution of each mirror to the total flux, in contrast with the continuous models which consider the whole surface of the heliostats field as being a reflector (see for example [11, 16, 25]). This model, constituting the basis of the program SOLA, was elaborated to study the thermal shocks in the receiver of a HFT. That is why, the program was built for this purpose without taking into consideration some perturbation phenomena, which 
decrease the intensity of the concentrated light beam. Consequently, we neglected both the effects introduced by the errors of the mirror surface manufacturing and the heliostats tracking errors, studied by us in detail in other papers $[27,28]$. Also, we deliberately neglected the heliostats shading and blocking by the neighbouring mirrors, as well as the semi-darkness area at the image edge, determined by the reflection of the whole solar disk on the mirror outline. Then, we considered that the atmosphere by which the reflected beam is being propagated does not introduce disturbing effects and all the heliostats are active. These simplifications give the program a greater operativity, with the simultaneous fulfilment of the main objective, which does not require an extremely precise knowledge of the concentrated radiation distribution, but only the correct establishment of the maximum concentration levels. The previous assertions concerning the precision of the physical model will also characterize the results presented further on. But, in compensation, due to the simplifications introduced, we can analyse economically a greater variety of possible situations.

As said in the introduction, the influence of the astronomical and constructive parameters on HFT working mainly refers to three aspects. The indicators proposed to translate this influence generally differ from one author to another, and determine the calculation method. Further on, we briefly present the indicators chosen by us and the principles of the mathematical model.

The introduction of the parameters characterizing the heliostat orientation and the mirror image was made by particularizing to the case of plane reflection surfaces the general method proposed by Igel and Hughes [5]. Thus, a tangential plane and a sagittal plane were defined (Fig. 1). The tangential plane con-

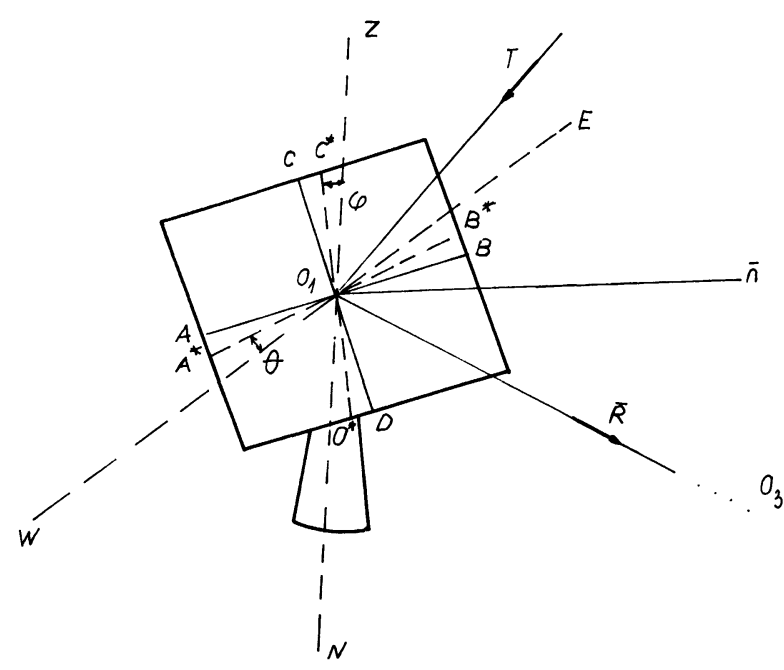

Fig. 1. - The angles $\theta$ and $\varphi$ obtained from the alignment condition. NZ-the Nadir-Zenith direction; EW-the horizontal East-West direction. AB, CD-the mirror symmetry axises; $\overline{\mathrm{I}}$-the main incident ray, $\overline{\mathrm{R}}$-the main reflected ray, $\bar{n}$-the normal in the mirror $\mathrm{O}_{1}$ centre. $\mathrm{O}_{3}$-the optic centre. tains the focal centre and the nadir-zenith direction which passes through the point $O_{1}$. The sagittal plane contains the focal centre and the east-west direction which passes through $\mathrm{O}_{1}$. The intersection of these planes with the plane mirror surface determines the straight lines $C^{*} D^{*}$ and respectively $A^{*} B^{*}$. We considered that the mirror orientation is specified by the angles $\varphi$ and $\theta$ formed by the straight lines NZ, $C^{*} D^{*}$ and respectively WE, $A^{*} B^{*}$. The mirror image is characterized by the dimensions $l_{\mathrm{IV}}$ and $l_{\mathrm{IH}}$, determined by reflecting in the image plane the segments $C^{*} D^{*}$ and respectively $A^{*} B^{*}$ (Fig. 2).

The determination of the angles $\varphi$ and $\theta$ was made by means of the tangential and sagittal planes, using the matric technique proposed by Wijeysundera [29] and imposing the alignment condition. Then, knowing the angles $\varphi$ and $\theta$ we determined the dimensions $l_{\mathrm{IH}}$ and $l_{\mathrm{IV}}$. Igel and Hughes [5] showed that the image form and dimensions depend on the heliostat operating mode. They noted that the specification of the mirror's surface orientation (in our case the specification of the angles $\varphi$ and $\theta$ ) does not univocally determine the image form, which can be modified by a mirror rotation around the normal in point $O_{1}$. Thus, a more efficient use of the heliostats can be performed by obtaining rectangular images with minimum dimensions [5]. These images are obtained for the minimum lengths of the segments $A^{*} B^{*}, C^{*} D^{*}$, namely $A B$, respectively $\mathrm{CD}$ (Fig. 1).

Further on, we will consider, according to Igel and Hughes [5], that the power reflected is uniformly distributed on the mirror image. We are defining the heliostat optical efficiency, the ratio between the real collecting area of the mirror, perpendicular on the direction of the incident radiation beam, and the image surface area in the focal plane. The optical

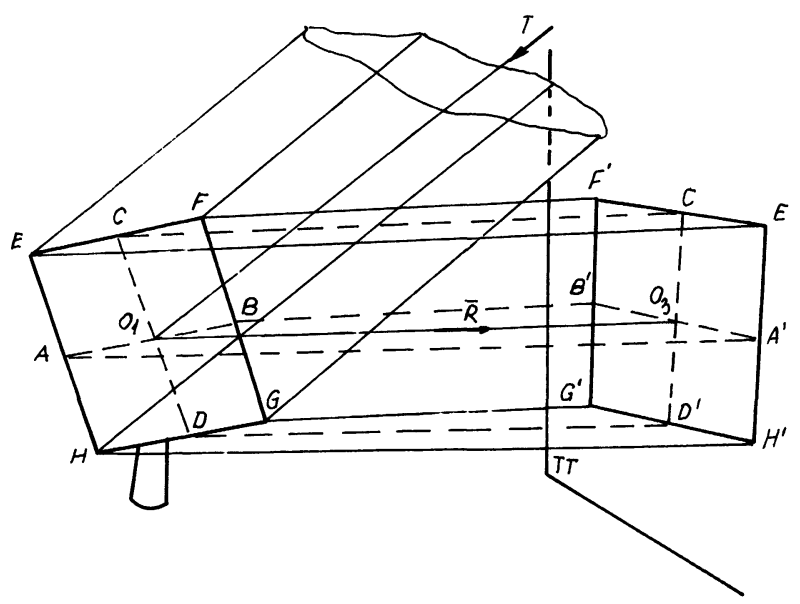

Fig. 2. - The dimensions $l_{\mathrm{IH}}$ and $l_{\mathrm{IV}}$ of the image, after the alignment and the rotation around the normal in $O_{1}$. $A B$ is an horizontal straight line, $\mathrm{CD}$ is a vertical one, $\mathrm{ABB}^{\prime} \mathrm{A}^{\prime}$ the sagittal section, $C D D^{\prime} C^{\prime}$-the tangential section; $l_{\mathrm{IH}}=\mathrm{A}^{\prime} \mathrm{B}^{\prime}, l_{\mathrm{IV}}=\mathrm{C}^{\prime} \mathrm{D}^{\prime}, \overline{\mathrm{I}}$-the main incident ray, $\overline{\mathrm{R}}$-the main reflected ray; $\mathrm{O}_{1}$-the mirror centre; $\mathrm{O}_{3}$-the optic centre; $\pi$-the image plane. 
efficiency is a measure of the concentration degree using only one heliostat. In case of plane mirrors, the optical efficiency is subunitary.

The intensity of the concentrated flux in a point $\mathbf{M}$ of the image plane is determined by the number and the optical efficiency of the mirrors which reflect in that point. We define the concentration degree in the point $\mathrm{M}, c$, as being a number showing how many times the concentrated radiation intensity is greater than the direct solar irradiance. The concentration degree in $\mathbf{M}$ can be determined by summing up the optical efficiencies of the heliostats which reflect in that point. The concentration degree is constant in ECR.

The characterization of the image by means of certain parameters, such as $l_{\mathrm{IH}}$ and $l_{\mathrm{IV}}$, was made by Igel and Hughes [5] in the case of spherical and toroidal heliostats. The image form and dimensions was also studied by Le Phat Vinh [8], in the case of parabolic heliostats, but using other specific parameters. None of the papers quoted in our references made a systematical approach on the influence of the astronomical and constructive parameters on the heliostats orientation mode. The angles $\theta$ and $\varphi$ have been introduced by us with this purpose. To characterize the form and the orientation of the curved mirrors, Igel and Hughes [5] defined a more complex system of angles. But these authors prefer to study systematically only the hourly variation of these angles. Various studies on the optical performances of only one heliostat, or of a whole field were developed by McFee [3], Igel and Hughes [5], Le Phat Vinh [8], Riaz and Gurr [16], Wei [25]. The indicators selected by each of these authors to characterize the optical performances differ more or less from our definition of the optical efficiency. A special attention has been paid to the study of the radiation distribution in the image plane or on the receiver surface (see the papers quoted in the introduction). Nevertheless, as far as we know, any systematic study on the mode in which the astronomical and constructive parameters influence the distribution of the concentration degree has been not yet presented. Moreover, certain studies analyse the concentrated radiation distribution directly on the surface of a special type of receiver (Riaz and Gurr [16], McFee [3], Blake et al. [1], Gintz et al. [30]), thus making difficult the results comparison.

2.3 THE COMPUTER PROGRAM. - The computer program SOLA [26] is organized on four modules with the following attributions :

(1) Reads the input sizes containing the geometric characteristics of the heliostats field, the latitude of the location, the day and the hour for which the calculus is made.

(2) Determines the astronomical parameters (hour angle, declination, azimuth, elevation) specific to the considered latitude, day and hour.

(3) Calculates, for each heliostat: (a) the co-ordi- nates of the centre $O_{1}$ within the heliostats field; (b) the distance from the heliostat to the optical centre; (c) the angles $\theta$ and $\varphi$ which check the heliostat alignment condition; (d) the co-ordinates in the focal plane of the image vertices; (e) the heliostat optical efficiency.

(4) Determines the total image and calculates the concentration degree distribution by the weighted superposition of all heliostats image.

\section{The technique of simulation.}

By using the program SOLA in the present paper we studied a heliostat field containing 64 rectangular mirrors (Fig. 3) and located at the latitude $\theta_{\text {lat }}=$ $45^{\circ} \mathrm{N}$. The initial values of the parameters, constituting the standard model, are presented in table I. The heliostats field consist of $N_{\mathrm{E}}$ mirrors rows in east-west direction and $N_{\mathrm{S}}$ mirrors rows on northsouth direction, respectively. In order to draw up the final conclusions as complete as possible we tested the following six heliostats $(i, j)$ (Fig. 3$)$ : $\mathrm{A}(1,1), \mathrm{B}(1,4)$, $C(1,8), D(5,5), E(8,1), F(8,8)$.

The image plane, of a rectangular form of A and B sides, is presented in figure 4. The optical centre is located at a height $H$ above the ground, and at a distance $f$ reported to the first east-west heliostats row (distance measured between the projection on the ground of the optical centre and the first east-west heliostats row). The image plane was covered with $21 \times 21$ points rows both in east-west and nadirzenith directions. The distance between the points rows, in both directions, is $p=0.25 \mathrm{~m}$. We analysed the following eight points $(\mathrm{I}, \mathrm{J}): \mathrm{A}(5,11), \mathrm{B}(6,11)$, $\mathrm{C}(7,16), \mathrm{D}(7,11), \mathrm{E}(11,17), \mathrm{F}(11,16), \mathrm{G}(11,15)$, $\mathrm{H}(11,11)$.

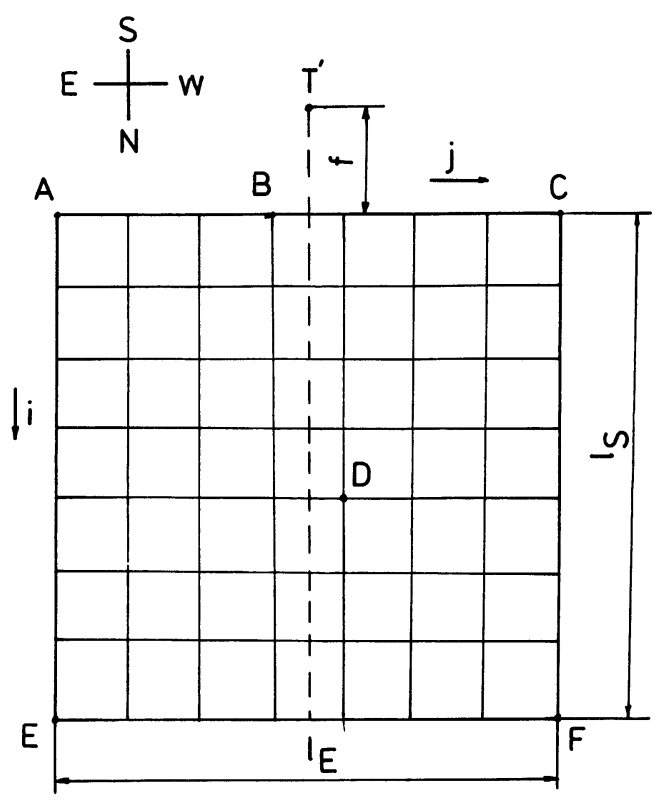

Fig. 3. - The heliostats field form and orientation. The position of the six tested mirrors. 
Table I. - The values of the main parameters during the simulation. All symbols defined in $\S 3$.

\begin{tabular}{|l|c|c|c|c|c|c|c|c|c|c|c|}
\hline \multicolumn{1}{|c|}{ Symbols } & $\theta_{\text {lat }}$ & $N_{\mathrm{E}}$ & $N_{\mathrm{S}}$ & $\mathrm{A}=\mathrm{B}$ & $h_{\mathrm{w}}$ & $n$ & $l_{\mathrm{E}}$ & $l_{\mathrm{S}}$ & $l$ & $f$ & $H$ \\
\hline Dimensions & degrees & - & - & $\mathrm{m}$ & hours & days & $\mathrm{m}$ & $\mathrm{m}$ & $\mathrm{m}$ & $\mathrm{m}$ & $\mathrm{m}$ \\
\hline $\begin{array}{l}\text { Value in the stan- } \\
\text { dard model }\end{array}$ & 45 & 8 & 8 & 5 & 8 & 152 & 20 & 40 & 2.0 & 10 & 20 \\
\hline Values for simu- & & & & & 6 & 62 & 25 & 20 & 1.5 & 20 & 10 \\
lation & & & & & 10 & 144 & 30 & 30 & 2.5 & 30 & 30 \\
& & & & & 12 & 192 & 40 & 60 & 3.0 & 40 & 40 \\
& & & & 15 & 242 & 60 & 80 & 3.5 & 50 & 50 \\
& & & & 16 & 290 & 80 & 100 & 4.0 & 60 & 60 \\
& & & & 17 & 332 & 100 & & & & 80 \\
& & & & & 18 & & & & & & 100 \\
& & & & & 19 & & & & & & \\
\hline
\end{tabular}

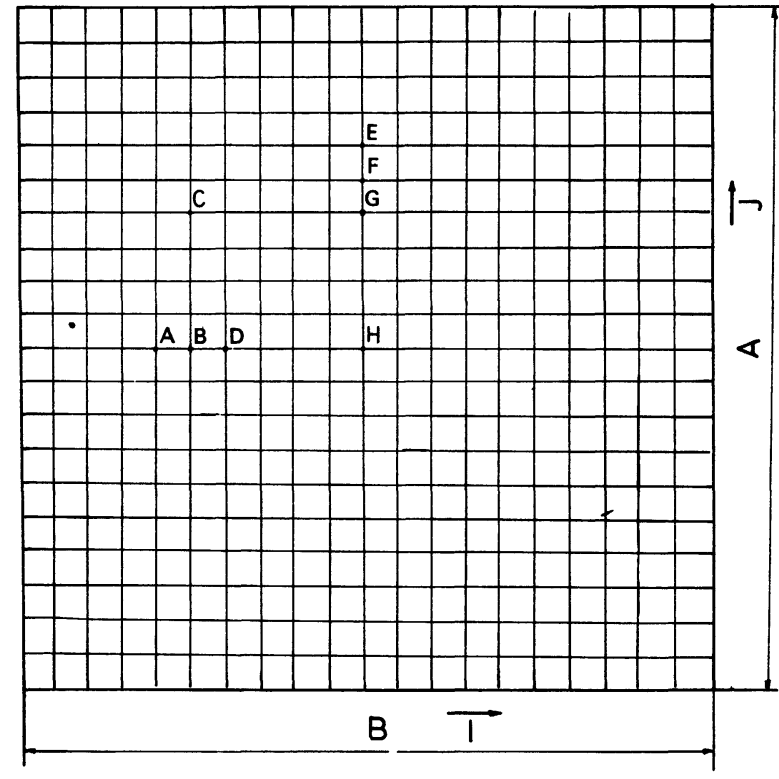

Fig. 4. - The image plane. The position of the eight tested points.

The technique of simulation was the following : we maintained constant the numerical values of the standard-model parameters, excepting the value of one parameter, which varied. In table I are presented all the values analysed during the simulation.

We tested the influence which have on the HFT working the following astronomical parameters : (1) the hour $h_{w} ;(2)$ the day, $n$, and the following constructive parameters : $(3,4)$ the distances between heliostats on the north-south and respectively eastwest directions; (5) the square mirror side dimension, $l ;(6)$ the tower height, $H$; (7) the distance between the tower and the heliostats field, $f$.
The HFT working was analysed from three points of view using the following indicators : (i) the mirror orientation was specified by means of the angles $\theta$ and $\varphi$; (ii) the heliostats optical performances were characterized by means of the image dimensions $l_{\mathrm{IH}}$, $l_{\text {IV }}$ and of the optical efficiency, $e$; (iii) the radiation concentration in the image plane was specified by means of the concentration degree, $c$.

\section{Results and discussions.}

The main object of our analysis was to provide useful information in HFT design. That is why, the results obtained studying the mirrors orientation were used to make certain remarks concerning the construction of the heliostat acting mechanism and the techniques of alignment. The study of the heliostats optical performances allowed us several conclusions related to the mirrors field form, the distribution of the covering factor of the field in the north-south and respectively east-west directions, the opportunity of using curved heliostats. Finally, analysing the distribution of the radiation within the image plane, we obtained certain observations concerning the receiver form and dimensions. At the same time we detached some information useful for selecting the receiver material and the daily mode of operation.

\subsection{ASTRONOMICAL PARAMETERS.}

4.1.1 The heliostats orientation. - We begin with the analysis of the hourly variation of the angles $\theta$ and $\varphi$.

The hourly variation of the angle $\theta$ has a similar behaviour for all the heliostats, decreasing monotonously from positive to negative values (Fig. 5). Regardless of the mirrors position this variation has the same daily deviation and the initial differences among the heliostats are maintained during the whole 


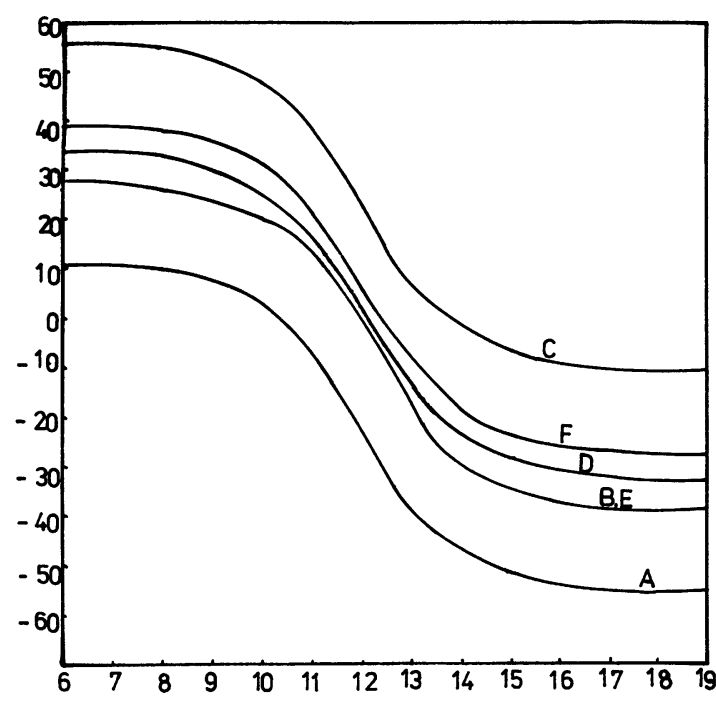

Fig. 5. - The variation of the angle $\theta$ (degrees), in ordinate, fonction of $h_{w}$ (hours), in abscisse, A, B, C, D, E, F-heliostats.

day. We can conclude that the device, which must correct the azimuth angle, may be built in a typified manner, under the condition of creating the possibility to perform an initial phase difference between heliostats, according to their location. The similar aspect of the hourly variation of the angle $\theta$, regardless of the mirrors position, justifies to take into consideration a programmed correction modality for the orientation in azimuth. This solution becomes even more applicable if the correction is made separately for groups of mirrors characterized by their position almost on the same horizontal line passing through the tower base. The previous assertion is argued by the similar hourly variation of the angle $\theta$ in case of the heliostats B and E, placed on the same line which passes through $T^{\prime}$ (see Fig. 3).

The greatest variation speed of the angle $\theta$ occurs, for all the heliostats, between $10 \mathrm{a} . \mathrm{m}$. and $14 \mathrm{p} . \mathrm{m}$. and it is of about 20 degrees $/ \mathrm{h}$, while the minimum speed, of about 2 degrees $/ \mathrm{h}$ is recorded at the beginning and at the end of the day. Consequently, the mechanism which correct the azimuth angle should have the possibility of continuously reducing and respectively adjusting the speed of revolution within a field of variation large enough. A detailed analysis of the proposed solutions for the heliostats acting mechanism, as well as the different modalities of mechanical equilibration was made by us in [21].

The determination of the angle $\theta$ hourly variation is rather laborious, especially in case of the fields with a great number of mirrors. When we are interested only in the maximum daily deviation of this angle, from figure 5 it can be noticed that it is sufficient, the analysis of the heliostats placed on the line passing through $T^{\prime}$ (see Fig. 3), shows the greatest angle with the north-south direction (the mirrors $\mathrm{A}$ and $\mathrm{C}$ in the case studied by us). Informations concerning the maximum daily deviation can be directly obtained by analysing the incidence angle of the main incident ray, which does not depend on the mirror's form and curvature. Indeed, closely analysing figure 14 from [5], we can remark that the daily maximum variation of the incidence angle does not exceed 60 degrees, for any of the tested heliostats. This variation is also specific for the values obtained by us, for the maximum deviation of the angle $\theta$.

The hourly variation of the angle $\varphi$ has the same aspect for all the heliostats, symmetrical as reported to the midday, increasing in the morning and decreasing in the afternoon (Fig. 6). As in the case of the angle $\theta$, the daily deviation of the angle $\varphi$ has in fact the same value, regardless of the mirror location. The initially existing differences among the heliostats are maintained during the whole day, also in this case. Consequently, it is possible to typify the construction of the device which corrects the elevation angle of the mirrors. The remarks, mentioned above, allow us to conclude that the programmed correction of the angle $\varphi$ is possible. In this context, it can be observed the fact that the heliostats symmetrically disposed as reported to the field north-south axis have the same hourly variation of the elevation angle. Further on, we shall take into account this aspect as well as the remarks mentioned as related to the angle $\theta$, in order to make some observations concerning the possibility of programming the orientation of the mirrors, both in azimuth and in elevation. The efficiency of this solution can increase if the orientation is made separately for groups of mirrors. The groups of mirrors can be associated two-by-two, symmetrically one another as reported to the field north-south axis. They will result by dividing the field in narrow rectangular portions with the long simmetry axis passing through $T^{\prime}$ (see Fig. 3).

The hourly speed of variation of the angle $\varphi$ is in fact the same for all the heliostats, having a constant

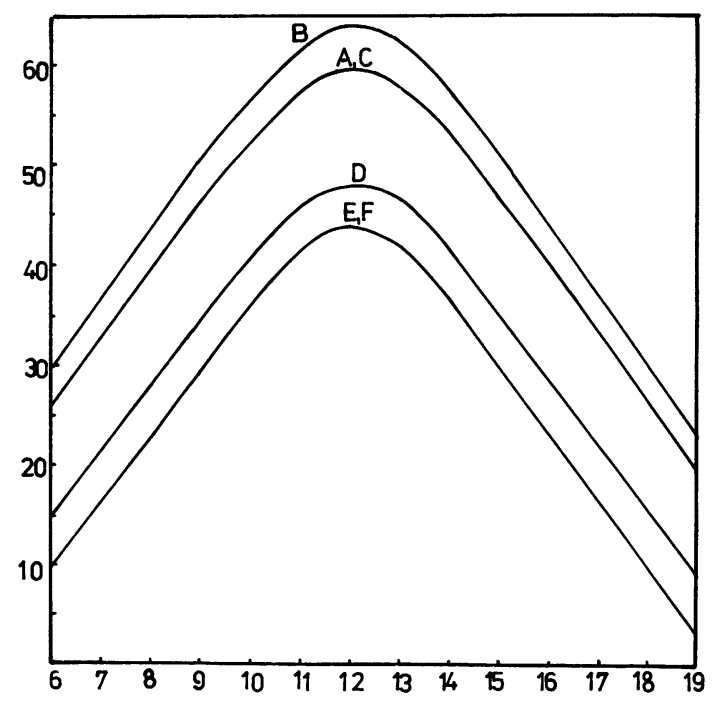

Fig. 6. - The variation of the angle $\varphi$ (degrees), in ordinate, fonction of $h_{w}$ (hours), in abscisse. A, B, C, D, E, F-heliostats. 
value of about 8 degrees/h up to 11 a.m. and respectively after 13 p.m. This fact determines certain differences concerning the constructive solutions of the mechanism which correct the angle $\varphi$ as compared to those intended to the azimuth correction [21].

If the designer is interested to know only the maximum range of variation of the angle $\varphi$ within the field it is enough to analyse the closest, and respectively the farthest heliostat from the tower (in our case the mirrors B and respectively E or F). Helpful informations concerning the daily deviation of the angle $\varphi$ can be found by analysing the daily deviation of the incidence angle, which is easier to be determined. Comparing figure 14 from [5] with figure 6 from the present paper it can be noticed that the ratio between the first and the second mentioned size is of about $1 / 2$.

The daily variation of the angles characterizing the mirrors orientation we are referring, has a stronger character in case of the azimuth angle. Indeed, for a given hour, the angle $\varphi$ is constant all through the year. This fact determines the constructive solutions of the elevation orientation mechanism not to be conditioned by the season in which the HFT will work.

The daily variation of the angle $\theta$ is similar for all heliostats, having a symmetrical aspect as reported to the middle of June and having a yearly deviation of about 10 degrees (Fig. 7). Consequently, it is necessary to perform an extra correction, beside that performed during the day, in case of using the solution of programming the azimuth orientation. As it can be noticed, this correction can be performed at longer time intervals in the spring and autumn and more often during the rest of the year.

The daily variation of the angle $\theta$ does not introduce new restrictions in designing the orientation acting mechanism as compared to those imposed by

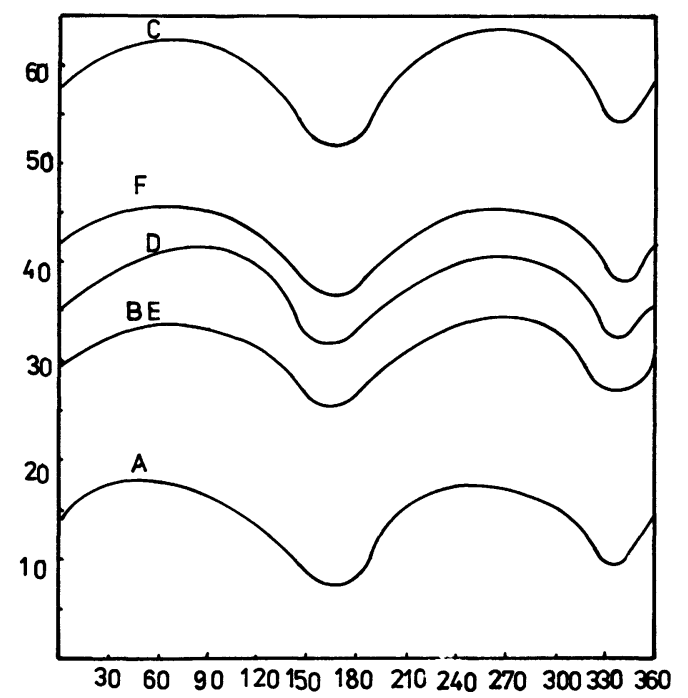

Fig. 7. - The variation of the angle $\theta$ (degrees), in ordinate, function of $n$ (days), in abscisse. A, B, C, D, E, F-heliostats. the variation during one day. The yearly maximum deviation can be determined by increasing the daily maximum deviation with about 10 degrees.

4.1.2 The heliostats optical performances. - First of all we are referring to the hourly variation of the heliostats images dimensions and their optical efficiency.

Excepting the heliostats A and C, the hourly variation of $l_{\mathrm{IH}}$ has for all the mirrors the same aspect, characterized by a rather too large deviation (about 25 per cent) and a maximum at the midday (Fig. 8). The heliostats $\mathrm{A}$ and $\mathrm{C}$ can be distinguished, besides their strong hourly variation, by the greater value than $l_{\mathrm{IH}}$ have during a half of the day. These two aspects are undesired and there are owed to the incorrect location of the two mirrors. In order to avoid such situations, a correct design should take into account during the heliostats location within the field simultaneously both their distance to the north-south simmetry axis and the distance from the tower. Indeed, it can be noticed that the heliostats $\mathrm{C}$ and $\mathrm{F}$ and respectively $\mathrm{E}$ and $\mathrm{F}$ do not have the same hourly variation of $l_{\mathrm{IH}}$, although they are disposed at the same distance from the north-south axis of the field and respectively at the same distance in the north of the tower. But the heliostats E and B placed about on the same line which passes through the point $T^{\prime}$ (see Fig. 3) has a similar hourly variation. These remarks allow us to conclude that, in order to make uniform during the day the performances of the heliostats, their location within the field should join within the limits of a certain circular sector with the centre in $\mathbf{T}^{\prime}$. In the case analysed by us, the heliostats $A$ and $C$ (and probably other neighbouring heliostats) were disposed outside the recommended circular sector.

Comparing the dimension $l_{\mathrm{IH}}$ of the heliostats $\mathrm{E}$ and $F$, it can be remarked, besides the similarity of the hourly variation reaching a maximum at midday,

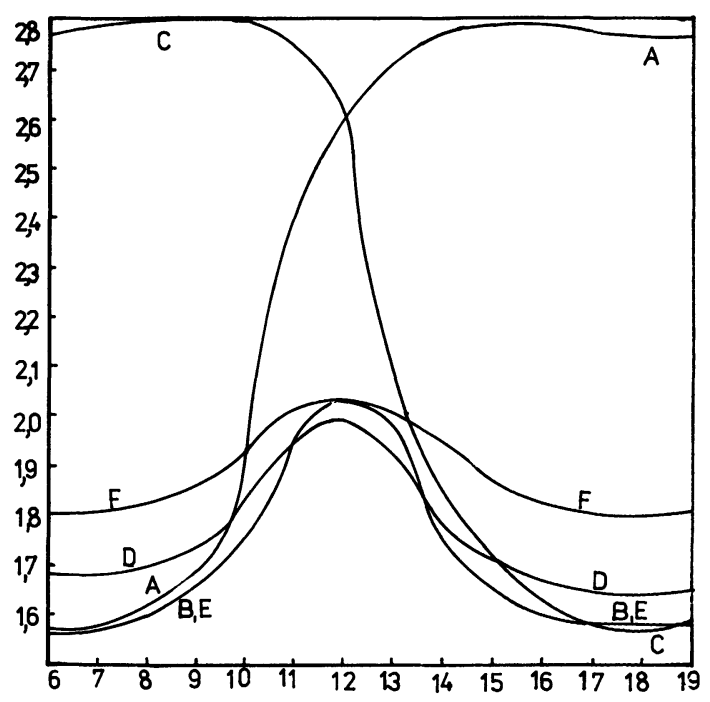

Fig. 8. - The variation of $l_{\mathrm{IH}}(\mathrm{m})$, in ordinate, function of $h_{w}$ (hours), in abscisse. A, B, C, D, E, F-heliostats. 
the reduction of the daily deviation of the western mirrors images. This fact can be corroborated with the hourly variations of the direct irradiance which also reaches a maximum at the midday of a clear day. Hence, in order to make uniform the concentrated power, it is recommanded to slightly set over towards the east the north-south symmetry axis of the field.

All the analysed heliostats have a small hourly variation of the dimension $l_{\text {IV }}$ (Fig. 9). This characteristic becomes more emphatic if the heliostat is farther from the tower. At the same time, the heliostat placed farther from the tower have smaller $l_{\mathrm{IV}}$ dimension. So, it is not recommanded to place the heliostats nearly the tower on the north-south direction. In the case analysed by us, the first east-west row of mirrors should be correctly located on the same straight line on which actually the heliostat $D$ is placed.

Comparing figures 9 and 8 it can be noticed that the $l_{\mathrm{IV}}$ dimension is greater than $l_{\mathrm{IH}}$ for all the mirrors. The difference is more evident for the mirrors closer to the tower. To obtain equilibrated images in the focal plane requires to use some technical solutions which may determine the reduction of the $l_{\mathrm{IV}}$ dimension. That is why, one of the common procedures consists in using toroidal mirrors. However, the performances of the curved heliostats which work all day long cannot exceed certain limits which we mention below, by comparing our results with the results obtained by other authors.

As long as the incidence angle is smaller, the image obtained by means of a parabolic heliostat is much smaller than that obtained using a plane heliostat, as shown by Le Phat Vinh [8]. The same is true for the case of toroidal heliostats. So, considering the figure 17 of Igel and Hughes [5] it can be seen that the daily minimum dimension of the image is of about 0.33 of the mirror side, while the same dimension in case of plane heliostats is superior to 0.75 of the

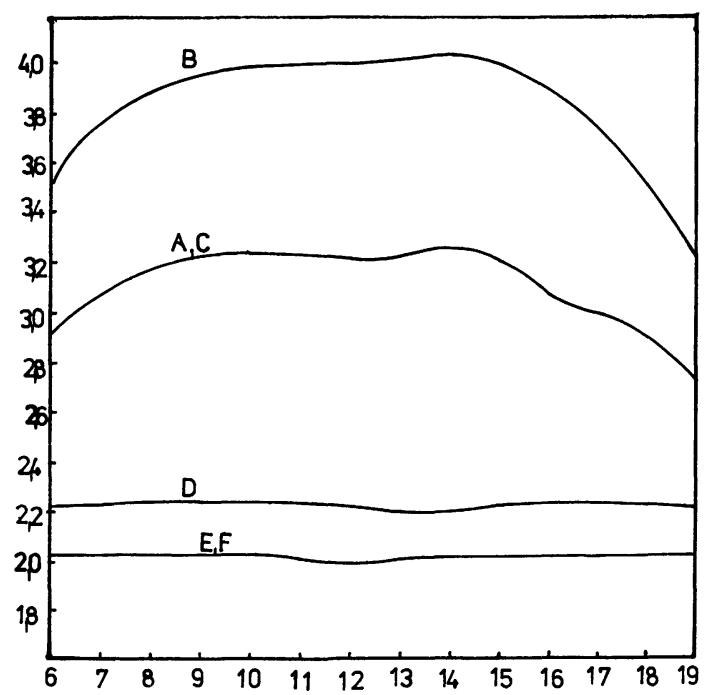

Fig. 9. - The variation of $l_{\mathrm{IV}}(\mathrm{m})$, in ordinate, function of $h_{w}$ (hours), in abscisse. A, B, C, D, E, F-heliostats. mirror side (Fig. 8 in this paper). The hourly variation of the image dimensions belonging to the curved heliostats is much stronger than that of the plane heliostats (see [8] and Fig. 8 from the present paper). This fact is owed to the astigmatism which causes the increasing of the curved heliostat image when the incidence angle increases. In case of toroidal heliostats for example, if the incidence angle is superior to 70 degrees the image in the focal plane is greater than that of a plane heliostat [5]. Analysing the results presented in figure 17 from [5] and our figure 8 we can conclude that, in spite of the astigmatism, the daily average value of the relative dimension of the curved heliostat image is smaller than that of the plane heliostats. Nevertheless, we think that the remarks mentioned above are in fact important impediments of the curved heliostats, which restrain the area within the field where they have an efficient and easily controllable work during the whole day.

The astigmatism determines the hourly variation of the image dimensions to have a different aspect in case of curved heliostats compared to the plane ones. This fact can be observed when comparing figures 17 and 18 from [5] and figures 8 and 9 from our paper. For the toroidal heliostats from [5] the image has minimum dimensions at midday, in contrast with the plane heliostats which generally at noon have an image of maximum dimension. If taking into account the hourly variation of the direct irradiance which has a maximum at midday, this fact differentiates certain characteristics of the HFT working, according to the type of the heliostats which are used. So, in contrast with the curved heliostats, the usage of the plane heliostats makes the concentrated power has a lower daily maximum value, but in exchange has a more uniform hourly variation.

The optical efficiency, from the point of view of our definition, is constant during the day for all the mirrors (Fig. 10). Its value depends on the heliostat location within the field, varying in the case analysed by us between 0.44 and 0.92 . The optical efficiency is smaller for the mirrors closer to the tower (A, B, C) and increases by increasing the north-south distance to the tower. The growth becomes more evident in the neighbourhood of the tower, as it can be observed by comparing successively the optical efficiency of the heliostats $\mathrm{A}$ and $\mathrm{D}$ and respectively $\mathrm{D}$ and $\mathrm{E}$. The heliostats placed symmetrically as reported to the north-south axis of the field (E, F and A, C) have the same efficiency, which decreases by increasing the distance between the mirror and the mentioned axis.

For the global characterization of the optical efficiency of all mirrors we defined following McFee [8] the heliostat D (placed close to the field centre) as being the reprezentative mirror. The global efficiency of the field analysed by us is then 0.87 , which can be compared to the average value 0.89 obtained at midday by Abatut and Achaibou (Fig. 8 from [15]). At the same time, we mention that owing to certain 


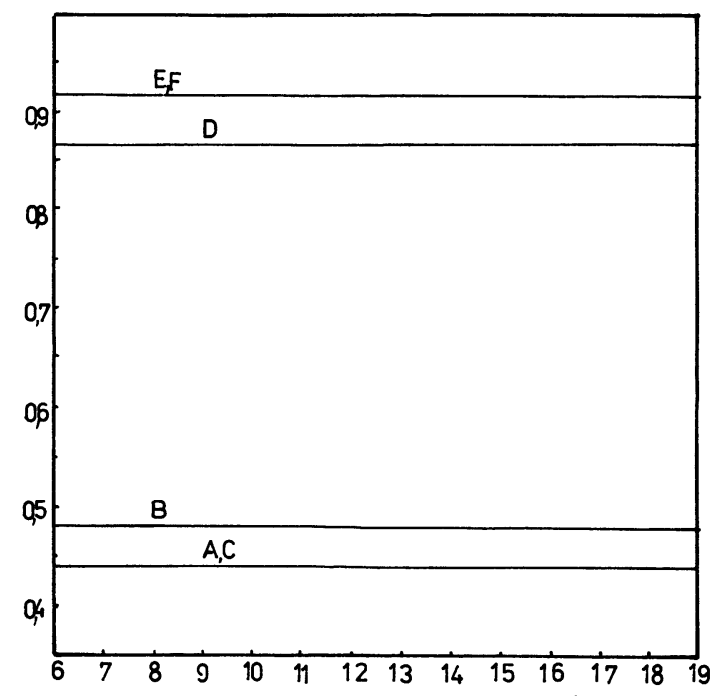

Fig. 10. - The variation of the optical efficiency, $e$, in ordinate, function of $h_{w}$ (hours), in abscisse. A, B, C, D, E F-heliostats.

corrective effects, the global efficiency in the case studied by Abatut and Achaibou decreases symmetrically around midday, with a maximum deviation of 20 per cent.

The results obtained by us allow to make evident the difference existing between the daily variation of the performances in case of using plane and respectively curved heliostats. So, owing to the astigmatism the parabolic heliostats performances from the eastern part of the field increase during the day, while the performances from the western part decrease (Figs. 3 and 4 from [8]). In case of toroidal heliostats the maximum performance are obtained at midday, when the astigmatism is minimum (Fig. 6 from [5]). The position of the heliostat $D$ from this paper is characterized by the same ratio between the distance to the tower and the tower height as the heliostat 18 from figure 16 of [5] has. Remark that between 10 a.m. and 14 p.m. the optical performances of these heliostats are quasi-constant ( 0.87 and 0.9 respectively). Beside this interval the performances of the toroidal heliostat decrease. Hence incomplete, the observations above suggest the idea that the daily working of the plane heliostats fields has a character easier to be controlled as compared to the case of using curved mirrors. Consequently, it can be found a certain incompatibility between the methods which determine the controllability improvement of the HFT performances and those which cause the improvement of their maximum performances.

Further on, we are referring to the daily variation of the optical performances. For a given hour both the vertical dimension of the image, and its efficiency are constant during the whole year, for all the heliostats. The optical efficiency of the whole field, whose value previously determined is 0.87 , is in agreement with the results obtained by Abatut and Achaibou [15], by using a more complicated physical model. Figure 8 from [15] shows a daily variation of the global optical efficiency in the range of 0.85 to 0.93 .

The daily variation of $l_{\mathrm{IH}}$ is rather reduced for all the heliostats, having a maximum deviation of about 15 per cent (Fig. 11). Excepting the mirror C, the images dimensions of the other mirrors are close one to another during the whole year. The range of variation of these dimensions has two minima, in June and December, when it decreases under 10 per cent of $l_{\mathrm{IH}}$, and two maxima, in February and September, when it reaches to about 20 per cent of $l_{\mathbf{l H}}$. It can be noticed that for each heliostat, the maximum and the minimum of $l_{\mathrm{IH}}$ are obtained in June and December and respectively in February and September. Consequently, during the whole year a natural compromise is achieved between the heliostats performances and the uniformity of their distribution within the field.

The daily variation of $l_{\mathrm{IH}}$ is stronger in the eastern part of the field (the mirrors A, E). This fact allows the remark that the slight east setting over of the field, previously proposed to increase $l_{\mathrm{IH}}$ uniformity, has as secondary effect the diminishing of the uniformity of this dimension during the year. But our results show the little importance of this negative effect.

The heliostats B and E, placed on the same line passing through $\mathrm{T}^{\prime}$ (see Fig. 3) have a similar variation of $l_{\mathrm{IH}}$ during the year. Consequently, this variation depends on the angle between the respective line and the north-south axis which passes through the receiver. The variation is smaller in case of greater angles (compare the mirrors A and E). To make uniform during the year the dimension $l_{\mathrm{IH}}$ can be obtained by the mirrors location close to the extremities of the circular sector which we defined above. Such a solution should accepted with caution, taking into account that it increases considerably the hourly variation of the field performances, as it has resulted from our previous analysis.

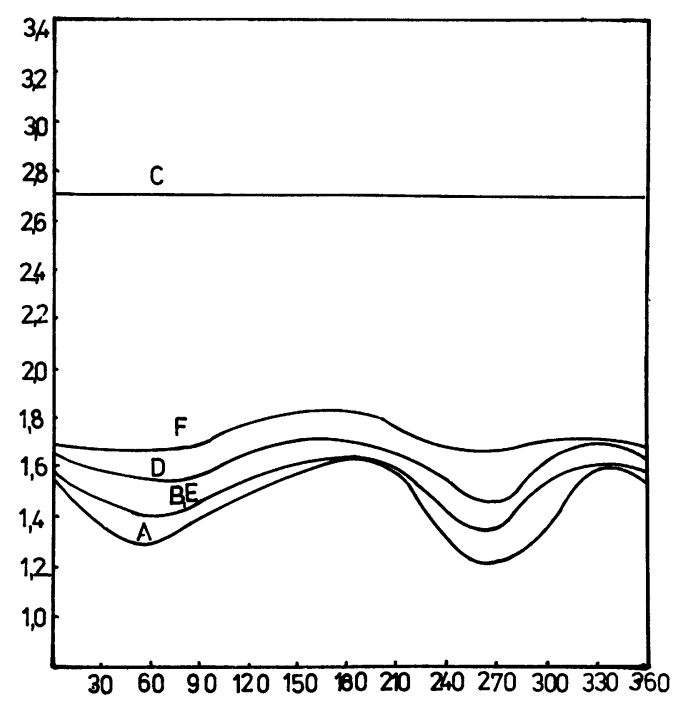

Fig. 11. - The variation of $l_{\mathrm{IH}}(\mathrm{m})$, in ordinate, function of $n$ (days), in abscisse. A, B, C, D, E, F-heliostats. 
4.1.3 The radiation concentration in the image plane. - The concentration degree is in fact constant all day long within ECR (the points $G, H$ ), where reflect all the field mirrors (Fig. 12) as well as in those regions of the image plane where light a small number of mirrors (the points A, B, E). An important hourly variation has the concentration degree in the marginal regions of ECR (the points $C, D, F$ ). The points from these regions are differentiated both from the point of view of the daily relative deviation of the concentration degree, with values between 100 and 300 per cent and regarding the aspect of the hourly variation of this parameter. By using figure 4 we will remark that the image plane region with strong hourly variation of the concentration also constitute the intermediate of the area between ECR (where $c \approx 50$ ) and the regions of weak concentration (where $c \leqslant 5$ ). This strong spatial variation of the concentration degree occurs on a small distance of about $0.5 \mathrm{~m}$. The previous remarks can be maintained as a rule if we refer to the distribution in the image plane of the concentrated flux, which can be obtained by multiplying the concentration degree with the direct irradiance value. The hourly variation of the irradiance (with a relative deviation within the interval between 9 a.m. and 15 p.m. always greater than 25 per cent) confers a maximum to the concentrated flux at midday in all the interior points of ECR, amplifying its hourly variation in the marginal region.

The main conclusion which results, is that the marginal region of ECR is characterized by strong temporal and spatial gradients of the concentrated flux. This fact justifies the special analysis of the fluids running lines on the receiver $[1,7]$ as well as the control of the fluid flow in case of transitory states [30]. At the same time, we remark that owing to the irradiance hourly variation the daily deviation of the concen-

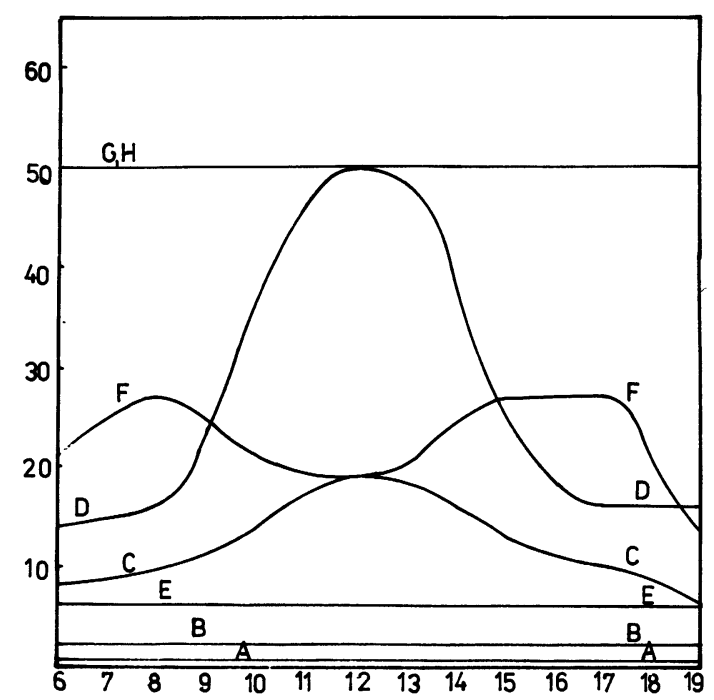

Fig. 12. - The variation of the degree of concentration, $c$, in ordinate, function of $h_{w}$ (hours), in abscisse. A, B, C, D, $\mathrm{E}, \mathrm{F}, \mathrm{G}, \mathrm{H}$-points in the image plane. trated flux exceeds 10 per cent within ECR and 25 per cent within the marginal region of ECR, values accepted by Blake $e t$ al. [1].

The concentration degree values are in fact constant all year long, in all the points of the image plane, excepting the marginal area of ECR (Fig. 13). Here the concentration degree has a daily variation between the minimum values of February and September and the maximum values of June and December. The yearly deviation is of about 100 per cent and overlaps the important daily deviation of the concentration in the marginal area of ECR. This fact should be taken into account both during designing the receiver and during its exploitation.

The most efficient period of work is within the interval April-August, when the concentration degree has higher values at ECR extremities and the direct irradiance is strong. During the whole year the area from the focal plane with strong concentration streches especially on its vertical dimension. But during the period April-August this area increases its horizontal dimension with about 30 per cent as it can be noticed from the location of the points $C$ and $D$ from figure 3.

Consequently, in case of the heliostats fields with plane mirrors of square form it is recommanded the cavity aperture should have the vertical dimension superior to the horizontal one (in the situation analysed by us their ratio is of about $6: 4$ ). The reduction of the aperture dimensions can be obtained by its inclination as reported to the image plane, with an angle which is not necessary to be modified during the year. The increase of the efficiency of solar radiation collection can be obtained by using certain superconcentration devices attached to the receiver on its lower and higher sides. To place such devices on the other sides is useful at most during the interval AprilAugust.

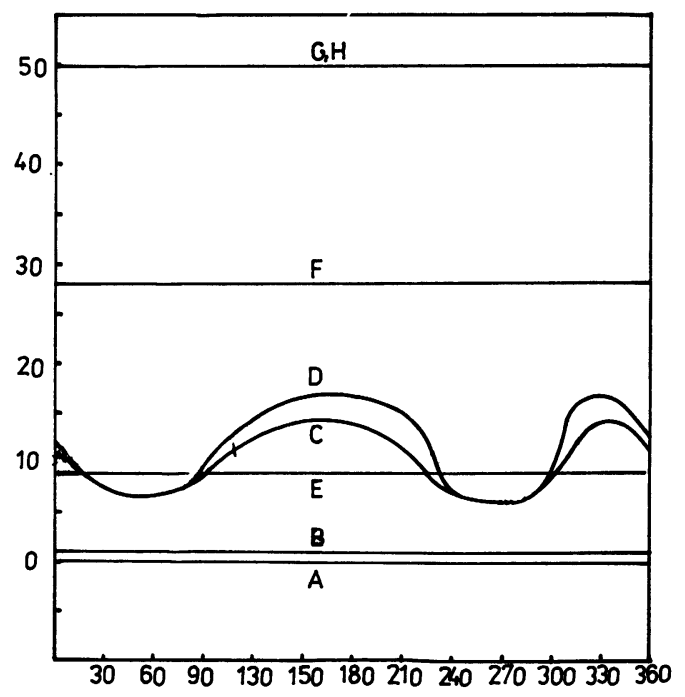

Fig. 13. - The variaton of the degree of concentration, $c$, in ordinate, function of $\boldsymbol{n}$ (days), in abscisse. A, B, C, D, E, F$\mathrm{G}, \mathrm{H}$-points in the image plane. 
4.2 CONSTRuCTIVE PARAMETERS. - Because the constructive parameters were presented in $\S 3$, we insist only on the definition of the distances between the heliostats in the east-west and respectively northsouth directions. These distances are given by the ratios between the east-west and north-south field dimensions $\left(l_{\mathrm{E}}\right.$ and $\left.l_{\mathrm{s}}\right)$ and the number of mirrors from an east-west and respectively north-south string $\left(N_{\mathrm{E}}\right.$ and $N_{\mathrm{S}}$ ). During the simulation $N_{\mathrm{E}}$ and $N_{\mathrm{S}}$ were maintained constant $\left(N_{\mathrm{E}}=N_{\mathrm{S}}=8\right)$, the dimensions $l_{\mathrm{E}}$ and $l_{\mathrm{S}}$ being variable between $20 \mathrm{~m}$ and $100 \mathrm{~m}$. Consequently, the distance between the mirrors varied in the range of $3 \mathrm{~m}$ and $14 \mathrm{~m}$, both in the eastwest and north-south directions. The variation of $l_{\mathrm{E}}$ and $l_{\mathrm{s}}$ was performed by maintaining in the same position the north-south axis of the field and respectively the first east-west string of heliostats. That is why, in the last case the mirrors A, B, C maintained constant their functional parameters.

First of all, this modality of modelation has the advantage of allowing the simultaneous study of all the mirrors placed on the same east-west (respectively north-south) string with the tested mirrors. Secondly, it can be noticed that it is possible a greater minution of the effect introduced by the field covering factor, which usually is not differentiated into its east-west and respectively north-south characteristics. Owing to the variation of $l_{\mathrm{E}}$ and $l_{\mathrm{S}}$ between $20 \mathrm{~m}$ and $100 \mathrm{~m}$ the covering factor varied between 0.35 and 0.07 and respectively between 0.66 and 0.13 .

4.2.1 The heliostats orientation. - The angles $\theta$ and $\varphi$ do not depend on the mirror dimension. However, we should mention that the heliostat size is determinant for the constructive solutions of the acting mechanism, as well as for its own orientation principle [21].

The angle $\theta$ does not depend on the tower height. But the angle $\varphi$ increases in all the analysed cases by increasing the tower height (Fig. 14). In case of small

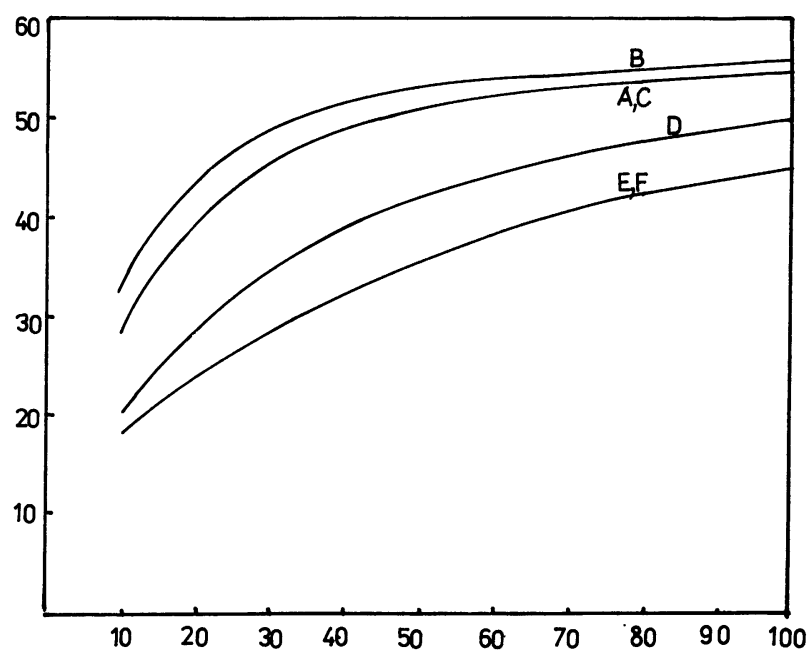

Fig. 14. - The variation of $\varphi$ (degrees), in ordinate, function of $\mathrm{H}(\mathrm{m})$, in abscisse. A, B, C, D, E, F-heliostats. heights the increase has a non-linear character which becomes more evident as the heliostat is placed closer to the tower. In case of higher heights the increase becomes linear, somehow stronger for the far heliostats and almost asymptotic in case of the heliostats close to the tower. As a results, the dispersion of the angle $\varphi$ values has a maximum for a tower height of $20-40 \mathrm{~m}$. So, it is obvious that, depending on the field form and dimensions, the programmed orientation of the heliostats raise some knotty problems for certain values of the tower dimensions placed in the region of the relatively small heights.

The deviation of the angle $\varphi$ within the tested interval is of about 25 degrees, approximately the same for all the mirrors. That is why the tower height influences the constructive solution of the acting mechanism.

Further on, we are referring to the influence that the distance between the tower and the field has on the heliostats orientation. The increase of this distance determines the increase of the angle $\theta$ in the eastern part of the field and its decrease in the western part (Fig. 15). The variation is more reduced in case of the mirrors far from the tower or close to the field northsouth axis and characterizes in our case only the mirrors A, B, C, which are unfavourably placed. That is why, we conclude that the strong decrease of $\theta$ values dispersion as a result of increasing the distance from the tower is especially characteristic for the fields incorrectly designed. This remark has two important consequences. First of all, the fact that in case of well-designed fields the distance between the tower and the first string of mirrors does not significantly influences the orientation procedure. The second consequence refers to the fields badly-designed which are characterized by a strong variation of the optical performances during the day (see Fig. 8). We remind the fact that the results from the figure 8 are obtained keeping in mind the hypothesis of a perfect orientation. At the same time, from what it was already

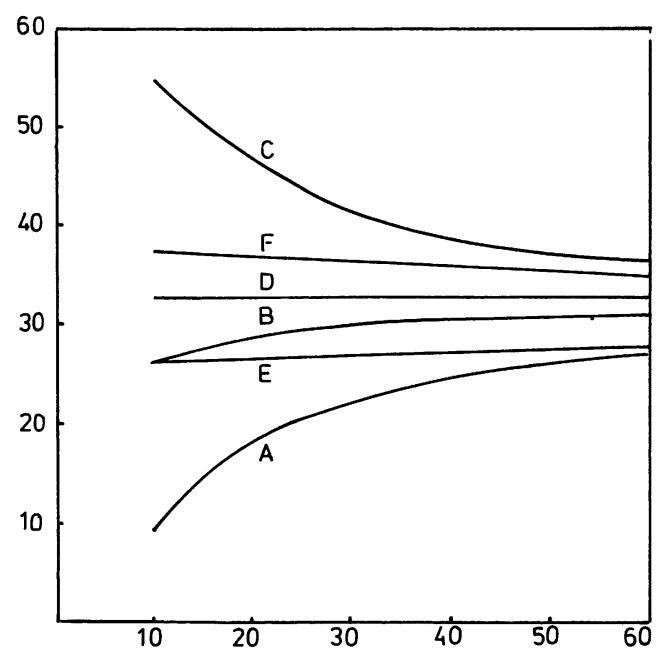

Fig. 15. - The variation of $\theta$ (degrees), in ordinate, function of $f(\mathrm{~m})$, in abscisse. A, B, C, D, E, F-heliostats. 
mentioned we conclude that, since the high value of the angle $\theta$ dispersion is specific to the fields badlydesigned, it is more difficult to obtain a good orientation for all mirrors for these fields. Consequently, it is expected that the non-uniform aspect of the optical performances during the day is even more evident in this case. The fact that the increase of the distance between the field and the tower may considerably diminish the secondary negative effect can be also remarked.

The angle $\varphi$ decreases by increasing the distance between the tower and the field, more evident as the mirrors are placed farther from the focus (Fig. 16). The heliostats A, B, C, incorrectly located, have a strongly non-linear variation of $\varphi$, which deviation is of about 20 per cent, while the deviation characterizing the other mirrors is of 5 degrees maximum. A similar conclusion to that, referring to the angle $\theta$ can be drawn : in case of the well-designed fields the distance $f$ can vary within rather large limits without affecting the constructive solution of the acting mechanism.

It can be also remarked that increasing $f$, the dispersion of the values of $\varphi$ has a significant decrease, even if we take into account only the mirrors favourably placed. Consequently, in contrast with the case of the angle $\theta$, by increasing the distance between the tower and the field, the possibilities to assure a good programmed correction of the angle $\varphi$ increase.

The heliostats orientation is influenced in a rather small measure by the north-south distance between the mirrors. So, the dependence of the angle $\theta$ is different according to the position of the mirror within the field (Fig. 17). The heliostats close to the north-south axis (mirror D) are very littly influenced compared to the farther mirrors which are stronger and influenced differently, according to their position in the eastern or western part of the field (mirrors $E$ and F). But in all situations we observe that the heliostats symmetric to the north-south axis have symmetrical variation curves as reported to the variation curve of the heliostat $D$. The variation is stronger at short distances between the mirrors, when it is shightly non-linear, and it has a maximum deviation of 10 degrees.

It can be also noticed that increasing the northsouth distance between the mirrors the interval which contains the angles $\theta$ of all the heliostats decreases, fact which favours the programmed correction of the orientation. Because the limit values of this interval are given by the heliostats from the field east and west extremities, the increase of the north-south distance between the neighbouring mirrors has good results if it is performed especially in these zones.

Increase the north-south distance between the mirrors always leads to the decrease of the angle $\varphi$ values (Fig. 18) (the apparent constant values of the heliostats $\mathrm{A}, \mathrm{B}, \mathrm{C}$ is owed to the simulation conditions).

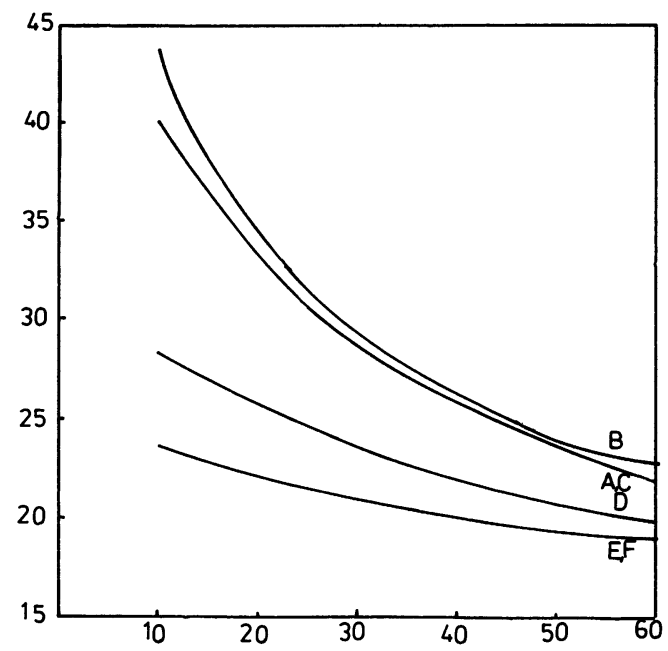

Fig. 16. - The variation of $\varphi$ (degrees), in ordinate, function of $f(\mathrm{~m})$, in abscisse. A, B, C, D, E, F-heliostats.

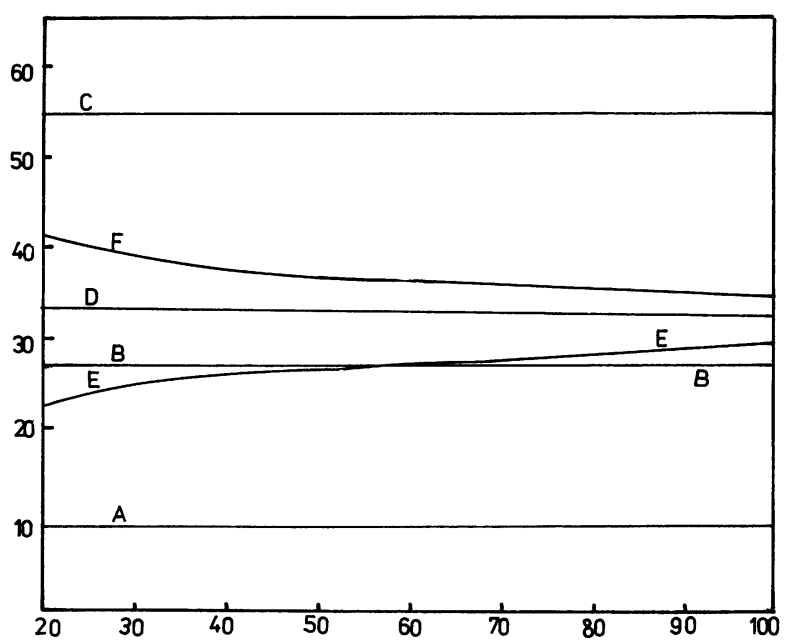

Fig. 17. - The variation of $\theta$ (degrees), in ordinate, function of $l_{\mathrm{s}}(\mathrm{m})$, in abscisse. A, B, C, D, E, F-heliostats.

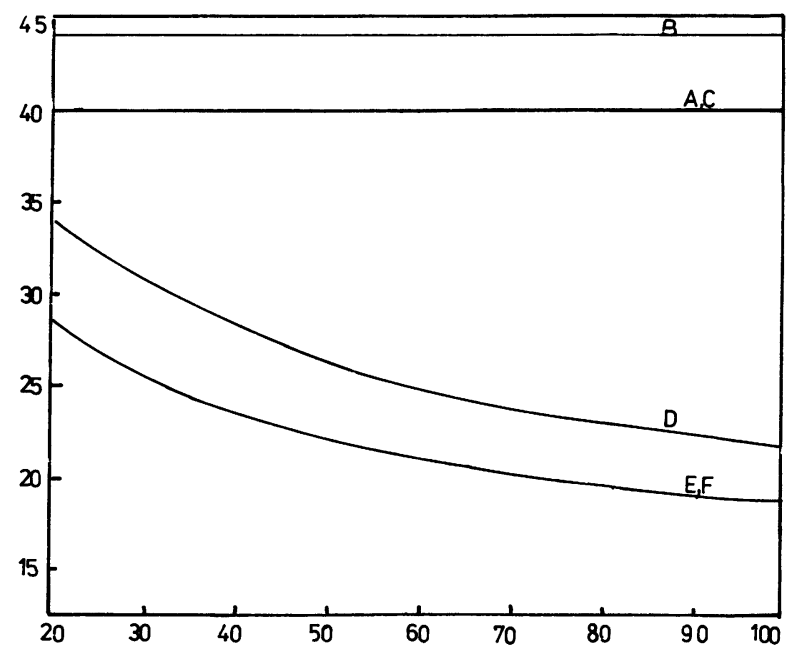

Fig. 18. - The variation of $\varphi$ (degrees), in ordinate, function of $l_{s}(\mathrm{~m})$, in abscisse. A, B, C, D, E, F-heliostats. 
The variation is similar in all the tested cases, a little stronger for the mirrors closer to the tower. The maximum deviation does not exceed 15 degrees.

The interval which contains the angles $\varphi$ of all the heliostats maintains actually the same value for any distance between the mirrors. Consequently, from this point of view there will be not restrictions concerning the principle of the orientation mechanism. However, we should mention that the utilization of the heliostats in the range of small $\varphi$ angles (a consequence of increasing the north-south distance between the mirrors) raise certain problems for the equilibration of the orientation mechanism [21].

We are now going to study the mode in which the heliostats orientation is influenced by the east-west distance between the mirrors. From this point of view the variation of the angle $\theta$ is different in the eastern part of the field (the mirrors B, E, A) as compared to the western part (Fig. 19) (increasing in the first case and decreasing in the second one). This variation has a slight non-linear character for all the heliostats and it is stronger when the distance between the mirrors is shorter. The relative deviation of the variation has a maximum value of about 15 per cent and it is more reduced in case of the mirrors close $r$ to the north-south axis of the field.

When design, the mechanical acting system cf the azimuth orientation should take into accourit the fact that the range of variation of the angle $\theta$ extends between 45 and 75 degrees. These values correspond to an east-west distance between the mirrors of $3 \mathrm{~m}$ and respectively $14 \mathrm{~m}$. Consequently, the mirrors spacing out makes difficult the adjustement of the acting mechanism, but it does not require to reconsider its principle. Also, by increasing the east-west distance between the mirrors it is remarked the accentuation of the initially differences concerning the orientation in azimuth. This fact can be observed from the aspect of the curves of the heliostats B and E. So, it is necessary to complete the data presented in $\S 4.1 .1$ concerning the programmed correction of the orientation in azimuth. Generally, this solution can easily be applied in case of shorter distances between the heliostats.

The east-west distance between the mirrors influences especially the orientation in elevation of the heliostats from the tower neighbourhood (A, B, C), for which the relative deviation of the angle $\varphi$ reaches to about 50 per cent (Fig. 20). The neighbouring heliostats placed in this field area have a greater differentiation of the elevation angle. Consequently, near the tower a special attention should be paid to the implication of the mirrors arrangement upon the practical modalities of orientation. From this point of view the programmed correction of the orientation can be better performed if the heliostats from the tower neighbouring are closer one to another, forming smaller groups.

In case of great distances from the tower the influence of the east-west distance between the mirrors is signi-

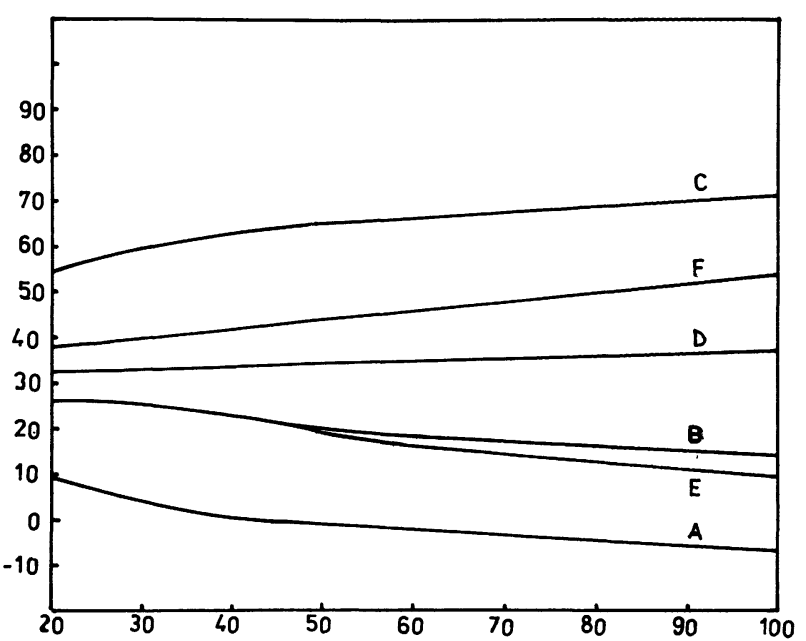

Fig. 19. - The variation of $\theta$ (degrees), in ordinate, function of $l_{\mathrm{E}}(\mathrm{m})$, in abscisse. A, B, C, D, E, F-heliostats.

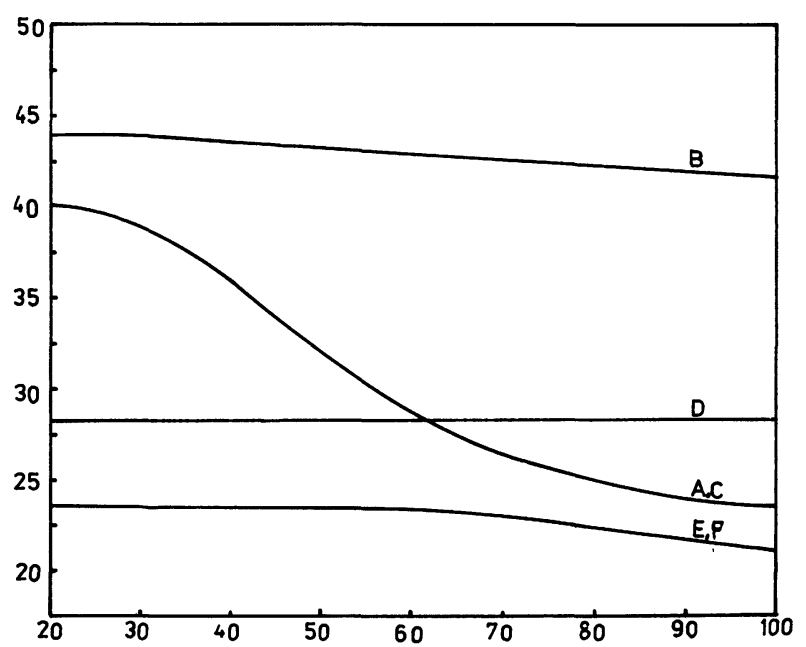

Fig. 20. - The variation of $\varphi$ (degrees), in ordinate, function of $l_{\mathrm{E}}(\mathrm{m})$, in abscisse. A, B, C, D, E, F-heliostats.

ficant only for the heliostats farther from the northsouth axis of the field. For instance, in case of the heliostats $\mathrm{E}$ and $\mathrm{F}$ the sensibility threshold is of about $10 \mathrm{~m}$. Consequently, in the areas far from the tower the orientation techniques are less dependent on the criteria of establishing the mirrors location within the field.

The interval which contains the values of the angle $\varphi$ for all heliostats is not sensible to the modification of the east-west distance between the mirrors. Consequently, changing the mirrors arrangement does not introduce any extra restrictions when design the acting mechanism which correct the elevation angle.

4.2.2 The heliostats optical performances. - First of all, we are going to analyse the modality in which the image dimensions depend on the mirrors size. 
Before beginning we mention that the optical efficiency defined by us does not depend on the dimension of the plane square mirror.

Owing to the plane surface of reflexion the horizontal dimension of the image increases linearly by increasing the mirror side. The most important variation occurs at the heliostats $\mathrm{C}$ and $\mathrm{F}$ located in the western part of the field. The values of the $l_{\mathrm{IH}}$ dimension are rather compactly grouped, fact which becomes more evident if we ignore the heliostat $\mathrm{C}$, unfavourable placed (see $\S 4.1 .2$ ). But the dispersion of these values increases when increasing the mirror side, owing to the stronger variation of $l_{\mathrm{IH}}$ in case of the heliostats far from the north-south axis of the field. The values become more dispersed especially owing to the heliostats incorrectly placed (in our case the mirror $\mathrm{C}$ ). This fact underlines the special importance of a well-design field in case of using great dimension heliostats.

By increasing the mirror side, the image vertical dimension increases linearly, a little stronger than in case of $l_{\mathrm{IH}}$ dimension (Fig. 22). The increase is strong for the heliostats close to the north-south axis of the field and also to the tower. The values of the $l_{\mathrm{IV}}$ dimension are less compactly grouped than those of the image horizontal dimension. At the same time, by increasing the mirror side the dispersion of this values increases more quickly in the first case. Both the high value of the dispersion and the strong increase are owed especially to the heliostats A, B, C, unfavourably placed. Consequently, the faulty design of the field has stronger negative implications in case of $l_{\mathrm{IV}}$ dimension.

Without taking into account the heliostats A, B, C, from figures 21 and 22 it can be noticed that by increasing the mirror side of about 2.6 times, the image dimensions increase only of about 2 times. So, in the case of well-designed fields the favourable effect of reducing the number of heliostats by increasing the mirror dimensions can be taken into account. To a certain extent, this solution reduces considerably the cost of the heliostats field increasing relatively less the tower and the receiver cost.

The dependence of the image dimensions on the mirror size can be diminished by curving the reflexion surface. So, among other solutions, there is the use of mirrors whose surface consists of facets which can be oriented separately $[5,13]$. This fact has the advantage to allow the adjustment of the mirror curvature according to the distance from the receiver, as Le Phat Vinh [8] proposes. At the same time it is also possible to reduce especially the dimension $l_{\mathrm{IV}}$ of the image (which is superior to $l_{\mathrm{IH}}$ ), by constituting toroidal surfaces of reflexion, as Igel and Hughes [5] suggest. But, besides certain sure advantages, the usage of the curved heliostats comes up against the disadvantage of the non-uniformity of performances during the day and the decrease of the controllability of the HFT working (as we remarked in $\S 4.1 .2$ ). That is

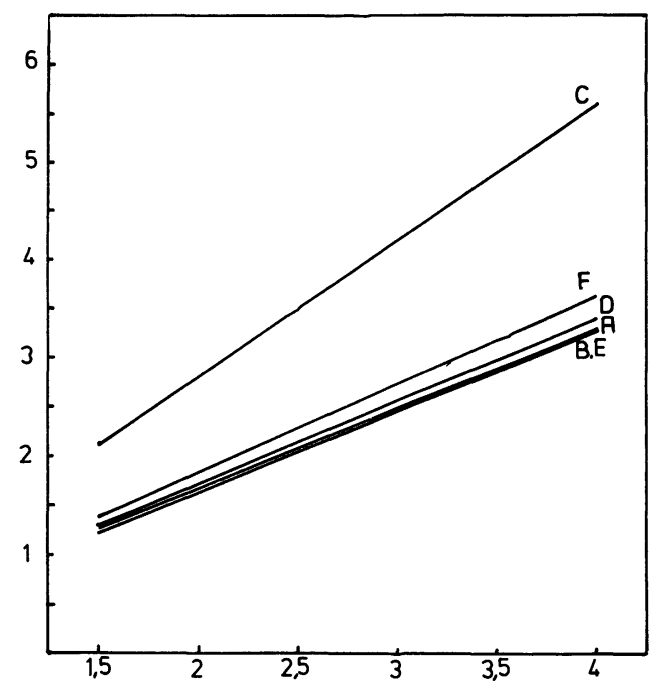

Fig. 21. - The variation of $l_{\mathrm{IH}}(\mathrm{m})$, in ordinate, function of $l(\mathrm{~m})$, the dimension of the square mirror side, in abscisse. A, B, C, D, E, F-heliostats.

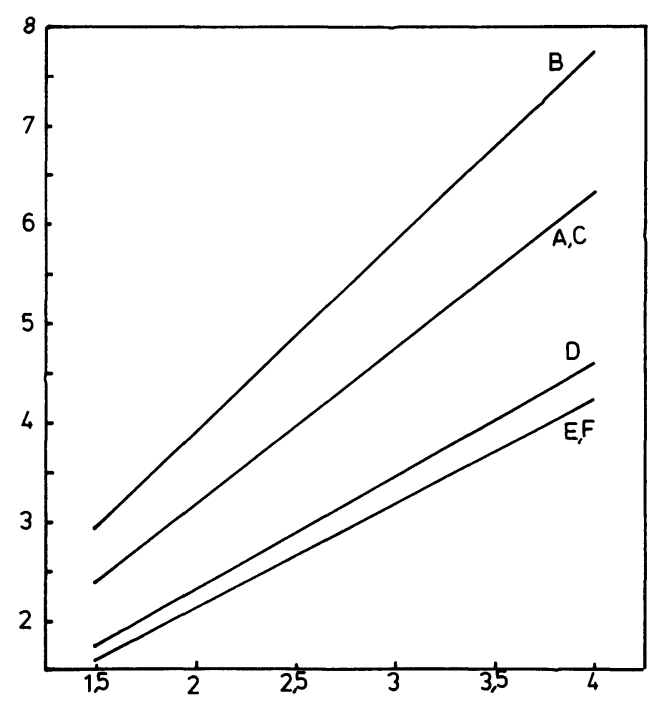

Fig. 22. - The variation of $l_{\mathrm{IV}}(\mathrm{m})$, in ordinate, function of $l(\mathrm{~m})$, the dimension of the square mirror side, in abscisse. A, B, C, D, E, F-heliostats.

why, it seems tempting to use rectangular plane heliostats with the horizontal side greater than the vertical one, as Malykoff and Aparissi [9] and Fourakis and Severson [12] proposed. This solution leads to the equilibration of the focal spot form, without losing the main advantages offered by the usage of the plane surfaces of reflexion.

Further on we are referring to the influence of the tower height on the heliostats optical performances. Our results show that the image horizontal dimension does not depend on the tower limit gauge. In exchange, the image vertical dimension increases by increasing the tower height, with a quasi-linear curve of variation in all the tested cases (Fig. 23). The increase is stronger 


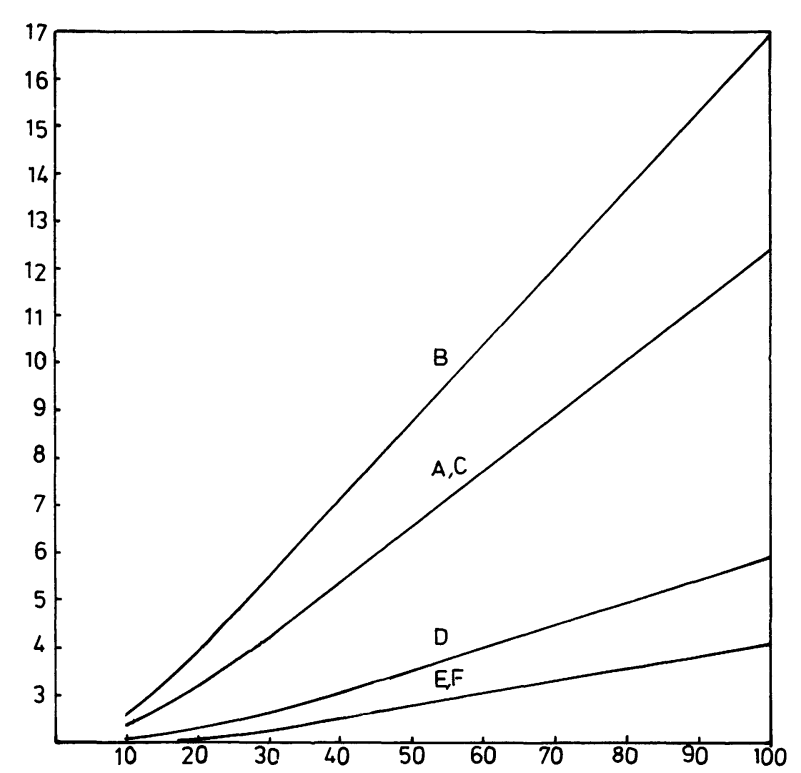

Fig. 23. - The variation of $l_{\mathrm{IV}}(\mathrm{m})$, in ordinate, function of $H(\mathrm{~m})$, in abscisse. A, B, C, D, E, F-heliostats.

for the heliostats A, B, C, placed in an unfavourable zone close to the tower. In this case the dimension $l_{\mathrm{IV}}$ increases of 5-6 times within the interval of analysis. The $l_{\mathrm{IV}}$ dimension of the heliostats $\mathrm{D}, \mathrm{E}, \mathrm{F}$, placed farther from the tower, increases of 2-2.5 times. It is worth to mention the fact that for a tower height of $60 \mathrm{~m}$ the $l_{\mathrm{IV}}$ dimension of the heliostat $\mathrm{D}$ exceeds two times the mirror side.

The dispersion of the values $l_{\mathrm{IV}}$ increases in case of greater heights of the tower, more strongly if the field is wrong-designed. Even by only taking into account the mirrors favourably placed, the deviation of the $l_{\text {Iv }}$ values increases of about 3 times by increasing $H$ from 40 to $100 \mathrm{~m}$.

The remarks above show that there are reasons, of exclusively functional nature, to place the receiver at not too high heights.

At the hour chosen for simulation the optical efficiency decreases by increasing the tower height, the more so as the heliostats are closer to the tower (Fig. 24). In all cases, the decrease is non-linear, but it has a differentiated aspect according to the mirrors correct or incorrect location. The first category of mirrors is characterized by a decrease with a rather uniform aspect while, for the second category the decrease is abrupt at low values of $H$ and tends to become stable by increasing the tower's height. Within the tested interval of heights the deviations of the optical efficiency are of about 0.45 and 0.6 for the correctly located mirrors and respectively for those incorrectly placed.

The dispersion of the optical efficiency values remains almost constant, with a slight maximum for tower heights in the range of 20 and $40 \mathrm{~m}$. If referring only to the unfavourably placed heliostats, it can be noticed that increasing the tower's height determines the uniformization at low values of their efficiencies.

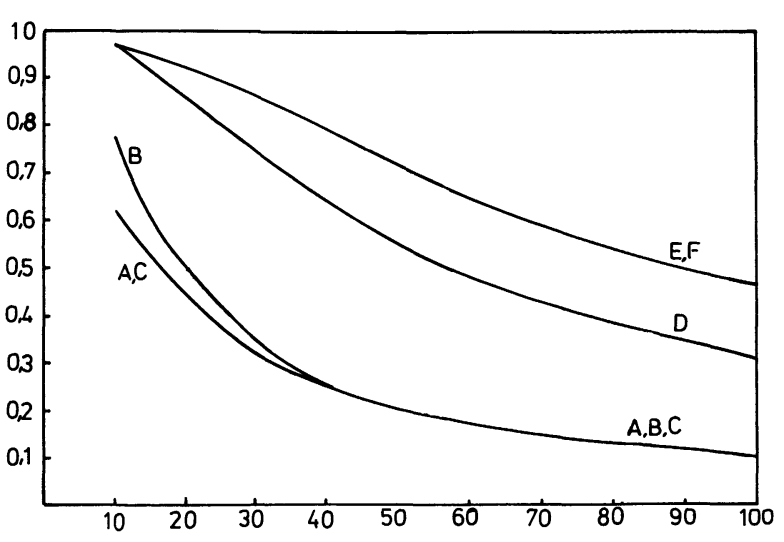

Fig. 24. - The variation of $e$, in ordinate, function of $H(\mathrm{~m})$, in abscisse. A, B, C, D, E, F-heliostats.

But, in exchange, for the other heliostats the dispersion of the efficiency values increases up to a certain height and then it tends to become stable.

The remarks above show that the increase of the tower height determines the depreciation of the HFT optical performances both owing to the decrease of the optical efficiency and owing to the increasing nonuniformity of this parameter's distribution on the field surface. But the shading and blocking of the heliostats by their neighbours, which we haven't taken into account, determines the tower height not to be less than a certain value. A compromise between the two contrary effects can be achieved by using receivers with vertical focal axis. In this case, the close heliostats will illuminate the receiver lower parts, while the far heliostats will reflect the radiation on its higher parts, thus resulting an uniformization of the optical performances on the field surface.

Coming back to the receivers with focal centre, the results presented in figure 24 confirm the opportunity of setting the heliostats field on a slightly incline slope on north-south direction, solution proposed by Blake et al. [1] and Abatut and Achaibou [15]. This fact determines, besides the reduction of the optical losses by shading and bloking, the diminution of the height difference between the farther mirrors and the focal centre, fact which increases their optical efficiency. For example, a slope of about 13.5 degrees on northsouth direction, close to that used by Blake et al., leads to a raising of about $12 \mathrm{~m}$ of the heliostats $\mathrm{E}$ and $F$, thus increasing their optical efficiency with 10 per cent.

The distance $f$ between the tower and the heliostats field has a rather strong influence on the mirrors optical performances. So, by increasing $f$, the image horizontal dimension slightly increases in the eastern part of the field and decreases in the western part (Fig. 25). At the hour chosen for simulation the image of the heliostat $\mathrm{C}$ (unfavourably located) has the strongest variation. It can be observed that a welldesigned field requires in our case a distance of at least $20 \mathrm{~m}$ between the first row of heliostats and the 


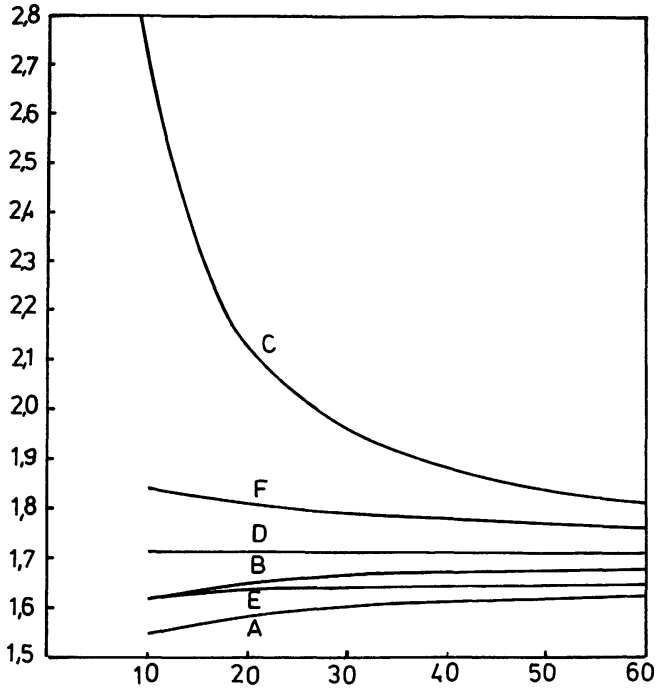

Fig. 25. - The variation of $l_{\mathrm{IH}}(\mathrm{m})$, in ordinate, function of $f(\mathrm{~m})$, in abscisse. A, B, C, D, E, F-heliostats.

tower, namely a distance comparable with the tower height.

The dispersion of the $l_{\mathrm{IH}}$ values decreases by increasing $f$. The variation is strong especially because of certain mirrors unfavourably placed and it is rather small in case of well-designed fields. Consequently, in this last case the variation of the parameter $f$ do not condition in a considerable manner the horizontal dimension of the receiver. Nevertheless, we should mention that, in case of long distances between the tower and the field, our analysis should be revised by taking into consideration certain disturbing phenomena which introduce errors proportional to the distance and which effect becomes important (see $\S 5)$.

The image vertical dimension decreases by increasing $f$, more rapidly for low values of this distance (Fig. 26). The strong non-linear character of $l_{\mathrm{IV}}$ variation in case of the mirrors $\mathrm{A}, \mathrm{B}, \mathrm{C}$, is an indicator of their unfavourable location. For the correct location of

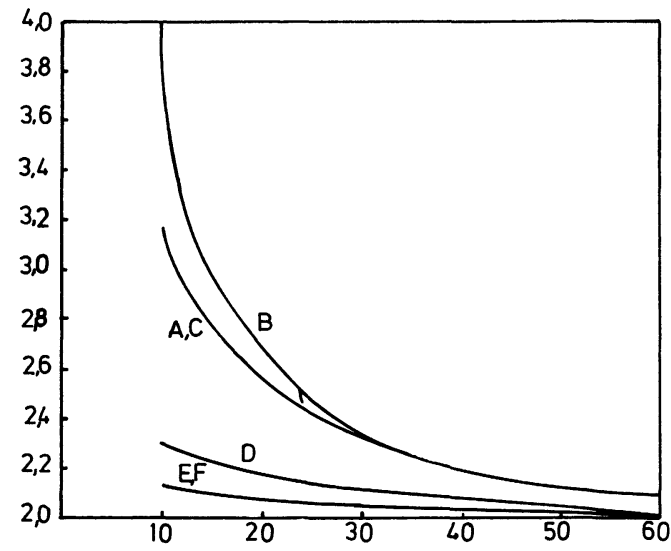

Fig. 26. - The variation of $l_{\mathrm{IV}}(\mathrm{m})$, in ordinate, function of $f(\mathrm{~m})$, in abscisse. A, B, C, D, E, F-heliostats. these mirrors $f$ should have at least $20 \mathrm{~m}$, namely the same value obtained by us when analysed the variation of $l_{\mathrm{IH}}$.

The dispersion of $l_{\mathrm{IV}}$ values is smaller than in case of $l_{\mathrm{IH}}$ and it decreases substantially when increase the distance between the tower and the field. In this context, it is worth to note the fact that the dispersion of the values $l_{\mathrm{IV}}$ from an east-west string of heliostats can be diminished by shifting to the north the mirrors close to the field north-south axis. The closer from the tower is the east-west mirrors row, the greater it is recommanded to shift. This fact results from observing the variation curves of the heliostats $A, B, C$.

In case of analysing the mirrors placed at long distances from the tower, the corrective effects mentioned when the variation of $l_{\mathrm{IH}}$ is studied should be taken into account. But their relative effect is more reduced in case of $l_{\text {IV }}$ dimension. First of all because the dimension $l_{\mathrm{IV}}$ is always superior to $l_{\mathrm{IH}}$. Secondly, from figures 25 and 26 can be remarked the fact that, in case of $l_{\mathrm{IV}}$, the increasing correction overlaps an even more stressed tendency of decrease, which diminishes its effect.

The optical efficiency increases by increasing the distance between the tower and the field (Fig. 27). The increase is strongly non-linear for the heliostats from the first east-west row and has a diminution of its intensity in case of the mirrors farther from the tower. The deviation of $e$ within the tested interval of $f$ is of about 0.45 for the heliostats $\mathrm{A}, \mathrm{B}, \mathrm{C}$ (incorrectly placed) and much more reduced, of about 0.1 , for the other mirrors.

The dispersion of the values of $e$ can be diminished by increasing the distance $f$. This reduction is insignificant if referring only to the mirrors $\mathrm{D}, \mathrm{E}, \mathrm{F}$, which permanently have high values of the optical efficiency. Consequently, in case of well-designed fields, by modifying the distance between the tower

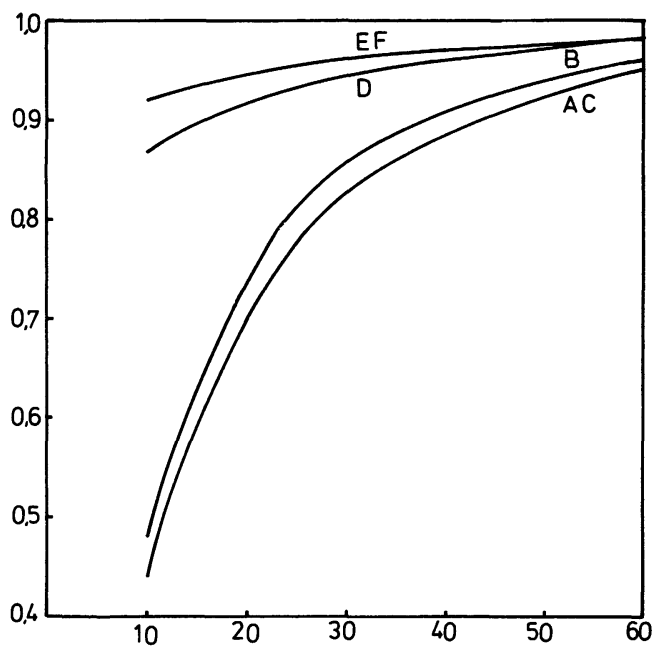

Fig. 27. - The variation of $e$, in ordinate, function of $f(\mathrm{~m})$, in abscisse. A, B, C, D, E, F-heliostats. 
and the field it can be obtained only minor influences on the HFT working uniformity.

We should mention that in case of great distances $f$ it is necessary to take into account the corrective effects mentioned during the analysis of $l_{\mathrm{IH}}$ and $l_{\mathrm{IV}}$. That is why, by increasing $f$ the optical efficiency will be gradually reduced, until it reaches a minimum followed by the depreciation of the performances. The corrective effect already mentioned determines the optical efficiency diminution and at the same time it also implies the increase of the HFT working uniformity. This remark is justified by the fact that the highest efficiencies characterize the heliostats far from the tower, for which the corrective effect is more important. Finally, we remark that in case of the toroidal heliostats Igel and Hughes [5] noticed the reduction of the optical performances if the distance between the mirrors and the tower increases. In contrast with the plane heliostats, in the case analysed by these authors the performances decreasing is monotonous.

Further on, we are studying the way in which the optical performances are influenced by the distance between the mirrors on the north-south direction. So, the image horizontal dimension is rather less influenced (Fig. 28). This fact can be observed especially in case of the mirrors close to the north-south axis of the field (heliostat D). The heliostats E and F, placed farther from the tower and respectively from the north-south axis of the field, have the most significant variation of $l_{\mathrm{IH}}$, which do not exceed 10 per cent within the interval of analysis. In the morning the variation is increasing in the eastern part of the field and non-linear decreasing in the western part, while in the afternoon the situation becomes reversed (see also Fig. 8). But the decrease effect is always dominating the increasing effect. Consequently, it is recommended that the north-south distance between the mirrors should be greater when the heliostats are far from the tower and especially in the north-east and north-west areas of the field. To illustrate this affirmation we are referring to the heliostats $F$ and $D$. Thus, in the case analysed by us, favourable effects are obtained if the north-south distance between the heliostat $F$ and its neighbours exceeds $7 \mathrm{~m}$. In case of the heliostat $D$, placed near the field northsouth axis, similar effects are obtained starting from a distance of $3 \mathrm{~m}$ between this mirror and its neighbours.

Generally, increasing the north-south distance between the mirrors determines the uniformization of the values of $l_{\mathrm{IH}}$ within the field, thus facilitating the design of the receiver.

The increase of the north-south distance between the mirrors has favourable effects, reducing the image vertical dimension (Fig. 29) especially for the mirrors placed close to the symmetry axis of the field (mirror $D$ ). Within the tested interval, the effect is nevertheless rather reduced, the relative deviation of $l_{\text {IV }}$ reaching maximum 15 per cent. The favourable effect has a greater intensity at small distances between heliostats

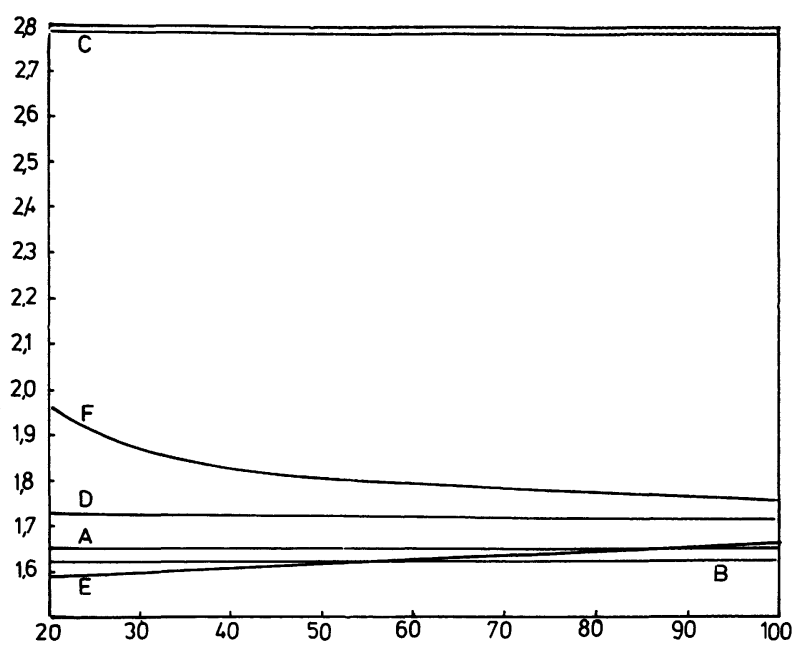

Fig. 28. - The variation of $l_{\mathrm{IH}}(\mathrm{m})$, in ordinate, function of $l_{\mathrm{S}}(\mathrm{m})$, in abscisse. A, B, C, D, E, F-heliostats.

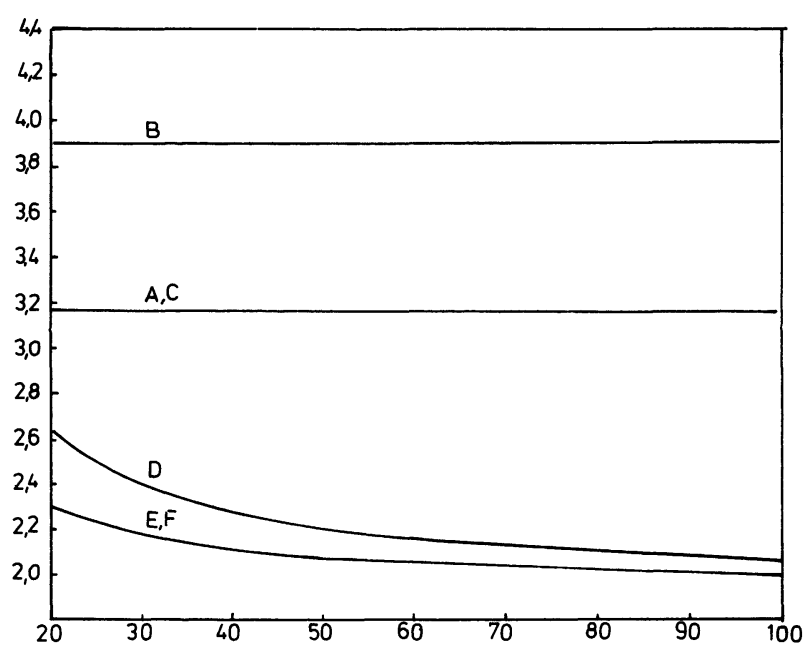

Fig. 29. - The variation of $l_{\mathrm{IV}}(\mathrm{m})$, in ordinate, function of $l_{\mathrm{S}}(\mathrm{m})$, in abscisse. A, B, C, D, E, F-heliostats.

and tends to a limit value. In case of the heliostats $E$ and $F$ this limit is in fact reached at distances greater than $7 \mathrm{~m}$ while in case of the heliostat $D$ the limit is reached to about $8.5 \mathrm{~m}$ distance. Hence, we conclude that special favourable effects are obtained when the north-south distance between heliostats is somehow greater near the field north-south axis.

The increase of the north-south distance between the mirrors determines the increase of the optical efficiency (Fig. 30). The increase has a deviation of about 0.15 within the tested interval and it is stronger in case the distance between the neighbouring heliostats is shorter. In the case analysed by us the values of $e$ tend to become constant for a distance between the mirrors longer than $10 \mathrm{~m}$.

It can be also noticed that, excepting the special case of the mirrors A, B, C, by increasing the distance between the heliostats the non-uniformity of the 


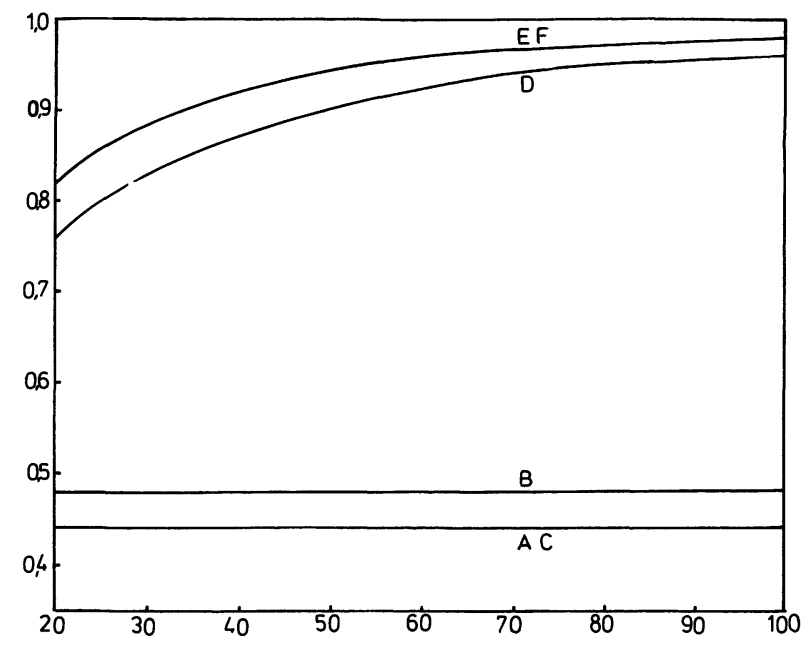

Fig. 30. - The variation of $e$, in ordinate, function of $l_{\mathrm{s}}(\mathrm{m})$, in abscisse. A, B, C, D, E, F-heliostats.

optical efficiency values distribution within the field increases. This effect is stronger in case of small distances between the mirrors.

The optical efficiency is conditioned not only by the heliostat image dimensions but also by the way in which the mirror intercept the incident radiation. This fact is evident if we study the different modalities in which the north-south distance between heliostats influences the parameters $l_{\mathrm{IH}}, l_{\mathrm{IV}}$ and $e$ of the heliostats D, E, F (Figs. 28, 29, 30). It can be drawn the important conclusion that only by arranging the mirrors distribution within the field cannot be obtained simultaneously both their more efficient usage and a proper form of the images in the focal plane.

The contrary effects mentioned above show that, according to the compromise between the technoeconomical criteria accepted during the design, the north-south distance between the mirrors cannot be increased over a certain limit. The existence of such a limit is also imposed by the fact that in case of great values of $l_{\mathrm{s}}$ the influence of certain disturbing phenomena which we neglected increases (see $\S 5$ ). In this context we should mention the results obtained by Abatut and Achaibou [15], using a more complex physical model. These authors show that increasing the north-south dimension of the field has for result the decrease of the ratio between the yearly collected energy and the surface of all the heliostats. But the HFT working is strongly differentiated according to the hour and day values. That is why we consider the analysis of the disturbing influences which appear in case of high north-south dimension of the field should be performed in detail by using an improved physical model.

We are referring now to the influence that the eastwest distance between heliostats has upon their performances. The variation of $l_{\mathrm{IH}}$ dimension is increasing in all cases and it has a quasi-linear aspect (Fig. 31). The dependence is stronger for the heliostats close

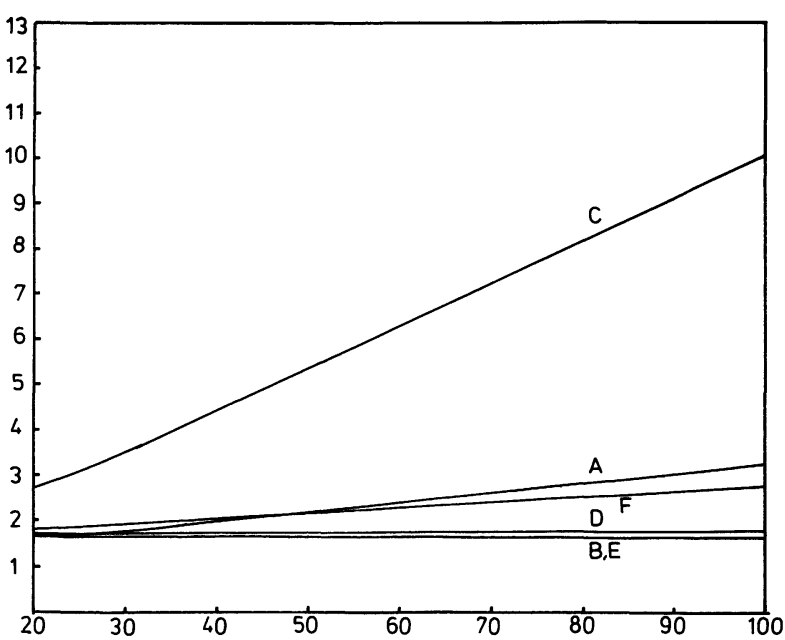

Fig. 31. - The variation of $l_{\mathrm{IH}}(\mathrm{m})$, in ordinate, function of $l_{\mathrm{E}}(\mathrm{m})$, in abscisse. A, B, C, D, E, F-heliostats.

to the tower and located in the western part of the field (during the morning) or in the eastern part of the field (during the afternoon) (see Table I and the curves A, C from Fig. 8). We should also remark that up to a distance of about $5 \mathrm{~m}$ between the mirrors, the values $l_{\text {IH }}$ for most of the tested heliostats are differentiated with maximum 25 per cent. The dispersion of the $l_{\mathrm{IH}}$ values increases continuously reaching to about 60 per cent for a distance of $14 \mathrm{~m}$ between the mirrors. The horizontal dimension of the receiving cavity aperture should be of about $2 \mathrm{~m}$ in the first case and $3 \mathrm{~m}$ in the second one, so depending strongly enough on the east-west distance between heliostats.

But the dependence of $l_{\mathrm{IH}}$ is more diminished for the mirrors placed in a region near the north-south axis of the field (the heliostats B, D), which increases its width when the distance from the tower increases. Thus, the region above constitutes that part of the field where the effects of blocking and shading can be avoided by increasing the distance between the mirrors, without an important depreciation of the image horizontal dimension.

The increasing of the east-west distance between the mirrors leads in all the tested cases to the reduction of $l_{\mathrm{IV}}$ dimension (Fig. 32). It can also be noticed that $l_{\text {IV }}$ variation is sensibly more reduced in case of great distances from the tower, being in fact negligible in the north half of the field. We conclude that any modification brought in this sense to the mirrors distribution in order to decrease the heliostats blocking and shading does not depreciate the vertical dimension of the image.

It is possible that by a certain arrangement of mirrors to obtain an almost complete uniformization of the heliostats images vertical dimensions. In the case analysed by us this fact occurs at an east-west distance of about $9 \mathrm{~m}$ between the mirrors. Consequently, the vertical dimension of the receiving cavity reduces significantly, from $3.15 \mathrm{~m}$ (in case the distance between the mirrors is $3 \mathrm{~m}$ ) to about $2.3 \mathrm{~m}$. 


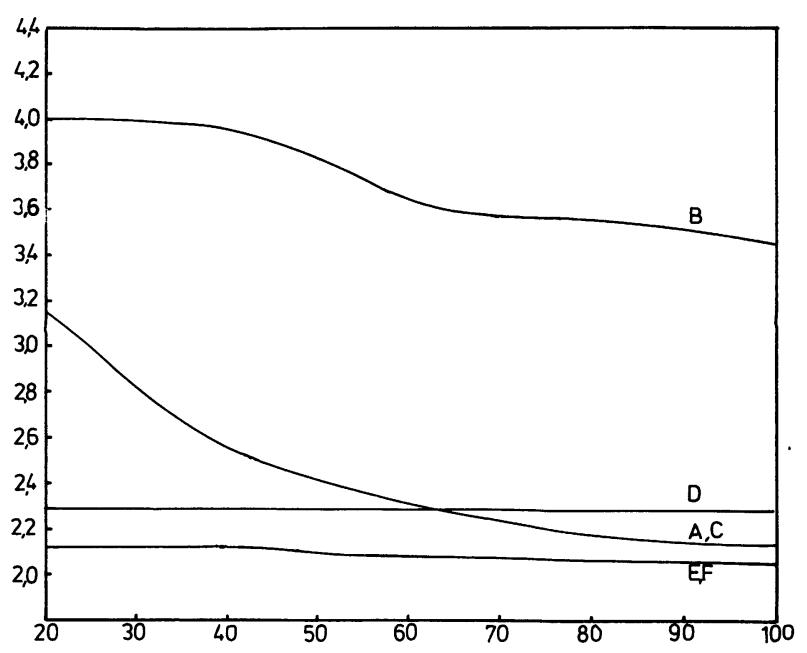

Fig. 32. - The variation of $l_{\mathrm{IV}}(\mathrm{m})$, in ordinate, function of $l_{\mathrm{E}}(\mathrm{m})$, in abscisse. A, B, C, D, E, F-heliostats.

Generally, the optical efficiency decreases when the east-west distance between heliostats increases (Fig. 33), the effect being stronger for the mirrors located farther from the field north-south axis (to be compared the mirrors A and B). Consequently, in this region it is possible to improve the optical performances by increasing the density of mirrors on the east-west direction. By comparing the heliostats $\mathrm{A}$ and $\mathrm{E}$ it can be remarked that the density of mirrors is recommended to be greater when the heliostats are placed much in the north of the tower.

At the level of the whole field the increase of the distance between the mirrors leads to a more pronounced differentiation of the optical performances, which continues to be symmetrical reported to the field north-south axis. This result, which characterizes the plane heliostats, can be compared with the results presented by Le Phat Vinh in figures 18, 19, 20 from [8], referring to the parabolic heliostats. In the case analysed by these authors, owing to the astigmatism, the increase of the field width leads to the accentuation of the asymmetry between the performances of the heliostats placed in the eastern part and respectively in the western part of the field. Just in the case of plane mirrors the results of Le Phat Vinh show that this asymmetry decreases by increasing the distance from the tower. The remarks above allow us to conclude that the restrictions which are conditioning the eastwest dimensions of the curved heliostats fields are stronger than those which are referring to the plane heliostats fields.

\subsubsection{The radiation concentration in the image plane.} - The increase of the heliostats size determines successively in the tested points the sudden jump of the concentration degree up to a maximum value, so that when the mirrors side reaches $3.5 \mathrm{~m}$ all the eight points belong to the ECR (Fig. 34). A short analysis of the numerical values and figure 4 shows that the influence of the mirrors dimension appears stronger at the

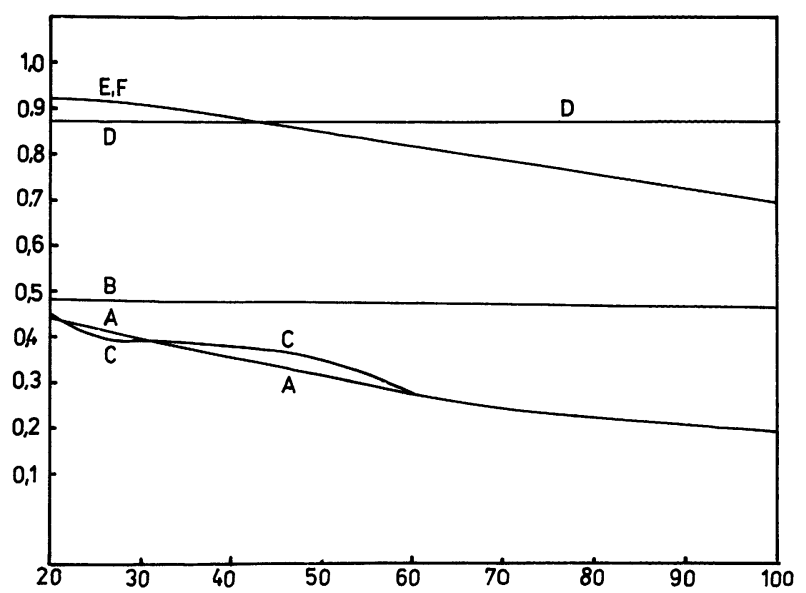

Fig. 33. - The variation of $e$, in ordinate, function of $l_{\mathrm{E}}(\mathrm{m})$ in abscisse. A, B, C, D, E, F-heliostats.

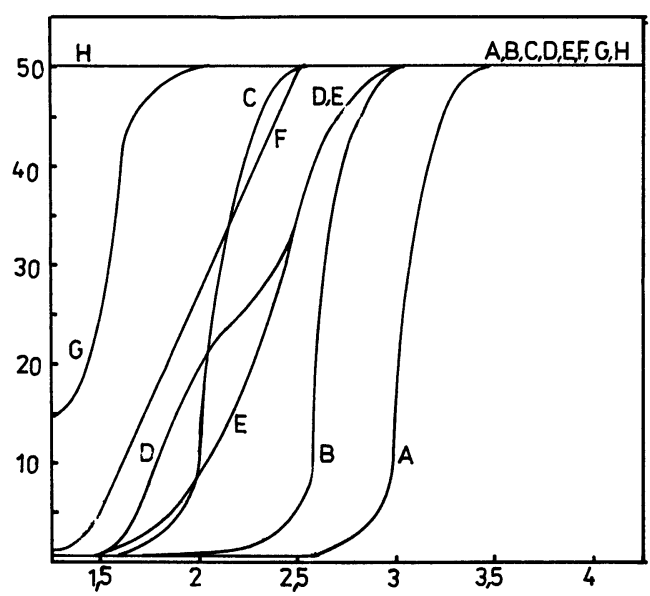

Fig. 34. - The variation of $c$, in ordinate, function of $l(\mathrm{~m})$, the dimension of the square mirror side, in abscisse. A, B, C, D, E, F, G, H-points in the image plane.

superior and inferior edges of the focal spot. So, in all the tested cases the region of strong concentration has the vertical dimension superior with about $1 \mathrm{~m}$ to the mirror side dimension and the horizontal dimension of the same size with the mirror side. Consequently, in case of plane mirrors of square form the receiving cavity aperture should be rectangular and placed with its longer side in vertical position.

The size of the plane heliostats does not influence the concentration degree from ECR but only the area of this region. At the hour chosen for simulation, in all the tested cases the vertical dimension of ECR exceeds the mirror side with about $0.5 \mathrm{~m}$ and the horizontal dimension of this region is inferior to the mirror side with about $0.5 \mathrm{~m}$.

What we mentioned above show that the ECR size increases proportionally to the mirror side while its neighbouring area has a width which is in fact constant. So, the increase of the mirror side determines a decrease of the weight which the area of strong 
spatial and temporary variation of the concentration has within the focal spot. Consequently, using heliostats of greater dimension has the advantage of a more uniform daily working of HFT.

By increasing the tower height the concentration has a different variation in the points of the image plane (Fig. 35). So, the concentration degree strongly decreases in ECR, varying between 55 and 20 . In exchange, the concentration increases in the neighbouring area, fact which determines the increase of the ECR surface. This fact occurs especially on the vertical dimension of the focal spot.

It can be also observed that the increase of the tower height has as result the concentration uniformization by the decrease of the difference which exist under this aspect between ECR and the rest of the focal spot. The tower height influence on the uniformization process is similar but it has a different weight in the lowest and highest parts and respectively in the sidewise parts of the focal spot. In the first case the vertical gradients of the concentration degree are great for small heights and decrease evidently by increasing the tower height. This fact can be ascertained following the values of the concentration degree in the points $\mathrm{E}, \mathrm{F}, \mathrm{G}$, when $H$ varies between 10 and $30 \mathrm{~m}$. In the second case the decrease of the horizontal gradients of $c$, which have values a little smaller than the vertical ones, occurs more slowly (see the points $\mathrm{D}$ and $\mathrm{H}$ ).

From the remarks above we can conclude that by increasing the tower height the maximum thermal performances decrease, but it increases the uniformity of the HFT working and decrease the thermal stresses within the receiver material. The compromise between these contrary effects determines the establishing of certain limits for the tower height, depending on the heliostats field dimensions and configuration (in our case the optimum height seems to be placed between 30 and $40 \mathrm{~m}$ ). The existence of an optimum height of the tower was remarked in another context also by Abatut and Achaibou [15]. Finally, we are mentioning that our results are in a certain measure directed by the simulation values of the day and the hour. That is why, the problem concerning the limits of the tower height should be detailed for a greater set of values of the two astronomical parameters and using an improved physical model.

By increasing the distance between the tower and the heliostats field, the concentration degree increases in ECR (the points $\mathrm{G}, \mathrm{H}$ ) and decreases monotonously in the other regions of the image plane (Fig. 36). Consequently, the focal spot decreases its dimensions, strongly in the highest and lowest parts (the points F, C, E) and more slowly on the sidewise parts (the point D), being able to be inscribed in a square. In exchange, the ECR continues to have a greater vertical dimension, as it results from the comparison of the concentration in the points $D$ and $G$, equally far from the focal centre $\mathrm{H}$. We conclude that in case of

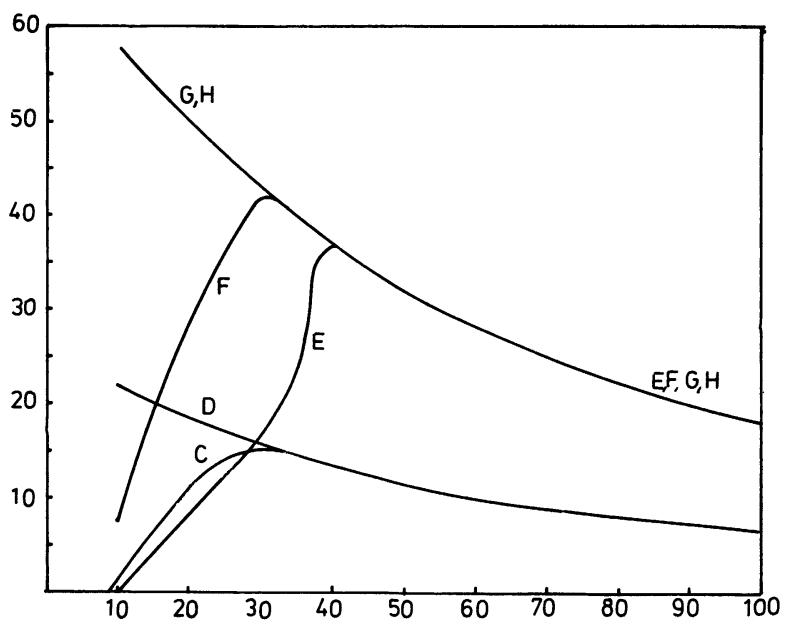

Fig. 35. - The variation of $c$, in ordinate, function of $H(\mathrm{~m})$, in abscisse. A, B, C, D, E, F, G, H-points in the image plane.

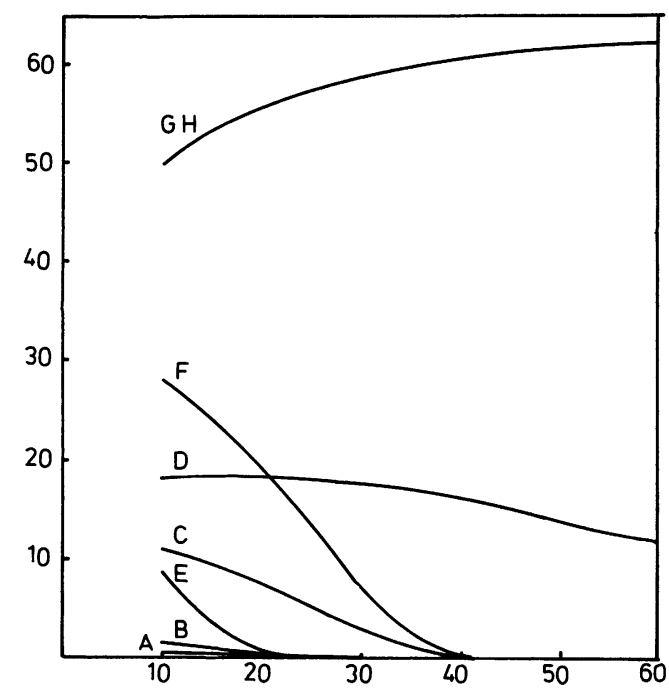

Fig. 36. - The variation of $c$, in ordinate, function of $f(\mathrm{~m})$, in abscisse. A, B, C, D, E, F, G, H-points in the image plane.

high values of $f$ the area from the image plane where the concentration has great daily variations it is distributed especially on the sidewise parts of the focal spot. Consequently, in these situations the design of the receiver aperture should take into account only the ideal vertical dimension of ECR and the collection of that part of the concentrated radiation which varies during the day can be made by means of certain sidewise super-concentrators.

The decrease of the vertical dimension of the focal spot is accompanied by the increase of the spatial gradients of the concentrated flux, which is much stronger on the vertical direction. The previous remark can be easily checked by comparing the values of $c$ in the points $\mathrm{E}, \mathrm{F}, \mathrm{G}$ and respectively $\mathrm{D}, \mathrm{G}$ for different values of $f$. We should take into account 
this fact both during selecting the receiver material and for establishing the lines of the working fluid.

The influence of the north-south distance between the mirrors on the concentration degree appears to be different in the ECR and respectively in the other regions of the image plane (Fig. 37). In the ECR the concentration degree increases from 45 to 53 by increasing the distance between the mirrors from $3 \mathrm{~m}$ to $14 \mathrm{~m}$. But the concentration decreases in the neighbouring region of ECR. This decrease is much more reduced in the ECR sidewise part as it results by the comparison of the points $D$ and respectively $F$.

We observe that for a distance between heliostats of about $3 \mathrm{~m}$ the point $\mathrm{F}$ is included in ECR together with the points $G$ and $H$. Consequently, the mirrors space out has as effect the decrease of the ECR surface, even more stressed on its vertical dimension. So, it is possible by a proper arrangement of the mirrors to be performed simultaneously both the increase of the maximum thermal performances and the equilibration of the form that the strong concentration region has. This last effect occurs owing to the much more stressed variation of ECR vertical dimension in comparison with its horizontal dimension.

At the same time we remark the fact that the increase of the north-south distance between heliostats determines the increase of the vertical spatial gradients of the concentration degree (respectively of the concentrated flux). So, the decrease of $c$ from 55 to 10 (the points $G$ and respectively $F$ ) takes place on a vertical length of about $0.25 \mathrm{~m}$ if the distance between heliostats is $14 \mathrm{~m}$ and on a length of $0.5 \mathrm{~m}$ (the points $\mathrm{G}$ and respectively $\mathrm{E}$ ) if this distance is $5.5 \mathrm{~m}$. In exchange, on the horizontal line the spatial gradients of $c$ are maintained in fact constant, with only a slight decrease (the points $\mathrm{D}$ and respectively $\mathrm{H}$ ).

What we mentioned above allow us to notice that in case of small distances between the mirrors the marginal area of ECR has a greater surface. Because the respective area is also characterized by important hourly variations of the concentration degree (see $\S 4.1 .3$ ) it is necessary in this case to grant special attention for establishing a proper HFT working mode during the day.

By increasing the east-west distance between the mirrors the concentration degree decreases within ECR (Fig. 38), varying in the tested interval between 50 and 40 (the points $G, H$ ). The same thing happens in the area from the superior and inferior edges of ECR (the points E, F). In exchange, on the sidewise edges of ECR the concentration degree increases (the points A, B, D).

Consequently, the mirrors space out has two different effects. Firstly, the maximum thermal performances decrease significantly. Secondly, the form of the focal spot equilibrates by increasing its horizontal dimension. Thus, in case of a distance of $14 \mathrm{~m}$ between the mirrors, the focal spot can be inscribed in a square of $3 \times 3 \mathrm{~m}^{2}$. At the same time the focal spot becomes

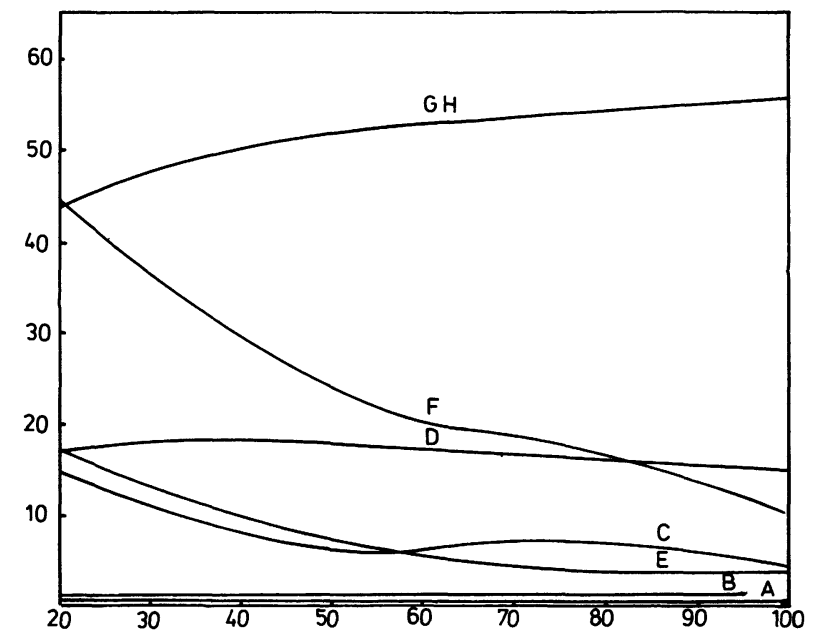

Fig. 37. - The variation of $c$, in ordinate, function of $l_{\mathrm{s}}(\mathrm{m})$, in abscisse. A, B, C, D, E, F, G, H-points in the image plane.

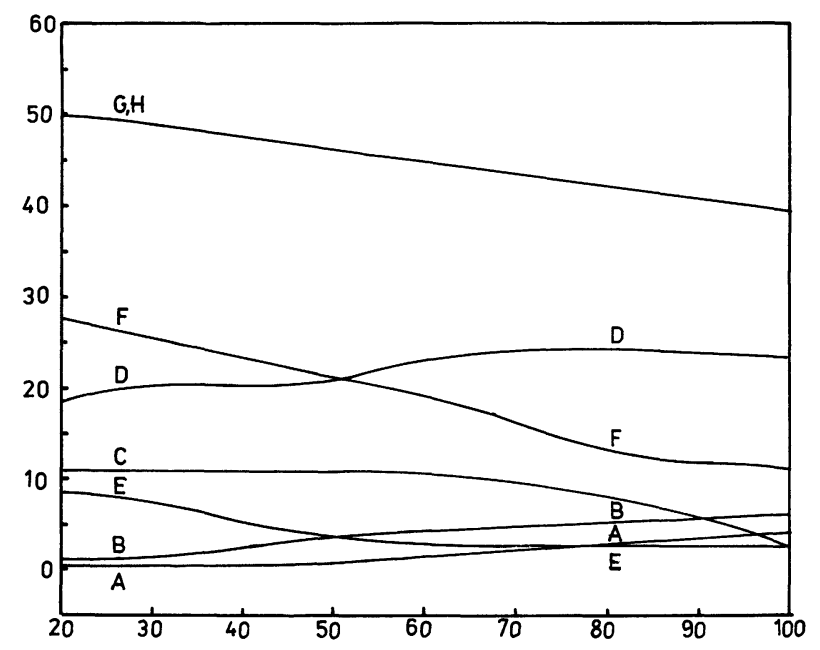

Fig. 38. - The variation of $c$, in ordinate, function of $l_{\mathrm{E}}(\mathrm{m})$, in abscisse. A, B, C, D, E, F, G, H-points in the image plane.

more diffuse, by the equalization tendency of the concentration degree within ECR and neighbouring areas.

Another favourable effect which should be mentioned is the decrease of the spatial gradients of the concentration degree. Thus, at an east-west distance between heliostats of $14 \mathrm{~m}$ the concentration degree decreases from 40 to 5 on a rather long distance of about $1 \mathrm{~m}$. As a result, the thermal stresses decrease, fact which facilitates the design of the fluid lines on the receiver surface.

\section{Remarks concerning the precision of the results.}

We will discuss at the level of principle the errors introduced by the simplifications used during elaborating the program SOLA. 
The deviation from the ideal case introduced by the imperfections of the reflection surface, as well as of the mirror form, do not affect the determined values of the angles $\theta$ and $\varphi$. But the dimensions $l_{\mathrm{IH}}$ and $l_{\mathrm{IV}}$ will be superior to those calculated by us. According Mc Fee [3] the correction to be made on $l_{\mathrm{IH}}, l_{\mathrm{IV}}$ from this point of view is negligible. However, we admit, following Riaz and Gurr [16] that the angle of dispersion introduced by the imperfection of the mirror is about $2 \mathrm{mrad}$. The distance between the heliostat $\mathrm{D}$ (the " mean " mirror of the field according $\S 4.1 .2$ ) and the optical centre is about $36 \mathrm{~m}$ (see Fig. 3 and the values $l_{\mathrm{E}}$ and $l_{\mathrm{S}}$ from the standard model, table I). So, it results an average underestimation of $l_{\mathrm{IH}}$ and $l_{\mathrm{IV}}$ around $0.07 \mathrm{~m}$. The deviation will be greater than the average deviation in case of heliostats $E$ and $F$, placed further from the tower, and smaller for the heliostats A, B, C placed closer the tower. The average deviations will be greater, up to doubling, in case of the results referring to the dependence of $l_{\mathrm{IH}}, l_{\mathrm{IV}}$ on the parameters $l_{\mathrm{E}}, l_{\mathrm{S}}, f, H$. The heliostat optical efficiency can be redefined supposing the radiation is uniformly distributed on the corrected image surface [5]. We are estimating the average correction to be made on the optical efficiency by taking into account that the dimensions $l_{\mathrm{IH}}, l_{\mathrm{IV}}$ were underestimated with $\Delta l_{\mathrm{IH}} \sim \Delta l_{\mathrm{IV}} \sim 0.07 \mathrm{~m}$. The corrected image surface area will be then larger than the area of the uncorrected image with about $l_{\mathrm{IH}} \cdot \Delta l_{\mathrm{IV}}+l_{\mathrm{IV}} \cdot \Delta l_{\mathrm{IH}}$. We assume an average uncorrected image area of the order $1.8 \times 2.2 \mathrm{~m}^{2}$ (see the heliostat $\mathrm{D}$ in Figs. 8, 9). In this case, results an average overestimation factor of the mirror optical efficiency around 1.07 (the overestimation factor is given by the ratio of the corrected and uncorrected areas). The average efficiency in the mirror field is about 0.87 (see § 4.1.2). Taking into account the overestimation factor, it will result a corrected average efficiency about 0.81 . The average overestimation of the optical efficiency is then 6 per cent. The overestimation factor will be greater for the heliostats $\mathrm{E}$ and $\mathrm{F}$, placed farther from the tower and smaller for the heliostats A, B, C placed closer to the tower. The concentration degree has a mean overevaluation of 6 per cent, according to what has precised when estimating the optical efficiency deviation.

The deviation introduced by the tracking errors to the values calculated by us refer especially to the angles $\theta$ and $\varphi$. We assume that the average tracking error is $3-5 \mathrm{mrad}[3,31]$, although there are orientation systems which allow errors under $1 \mathrm{mrad}[20,31]$. Using the reflection law, the range of variation for the angles $\theta$ and $\varphi$ can be determined, it is $\pm 0.5^{\circ}$. The influence of the tracking errors on $l_{\mathrm{IH}}, l_{\mathrm{IV}}$ (and consequently on the optical efficiency) is negligible [31]. Indeed, in case of short distances between the mirrors and the tower the tracking errors determine the same shift for all of the image points in the focal plane.
Supposing that the tracking errors of all the mirrors are equal and uniformely distributed around the optical centre [31], it results an average radial increasing of the total image about $0.06 \mathrm{~m}$ (we considered the case of the "mean " heliostat $D$ and a tracking error of $3 \mathrm{mrad}$ ). This increasing does not modify the concentration degree in the points B, D, F, $\mathrm{G}, \mathrm{H}$ placed much within the total image, but it is possible to cause an increasing (up to 10 per cent) of the concentration degree in the marginal points $\mathrm{A}, \mathrm{C}$, E. However, it must be taken into account the fact that there are situations when the tracking errors distribution is unisotropic [27], fact which may determine the total image decentralization as reported to the optical centre.

The angular finite dimension of the solar disk $(9.3 \mathrm{mrad})$ does not influence the values of the angles $\theta$ and $\varphi$. Repeating in the same way the calculus made for the evaluation of the deviations caused by the mirrors surface imperfections, it results an average underestimation of $l_{\mathrm{IH}}$ and $l_{\mathrm{IV}}$ around $0.32 \mathrm{~m}$. The heliostat optical efficiency shouldn't be redefined because the correction made does not modify the radiation distribution within the ideal image, but it introduce an additional area of semi-darkness. This semi-darkness zone increases the concentration degree in the points of the immediately neighbouring regions of the ideal image boundary. So, from this point of view the concentration degree is possible to be underestimated in the points $A$ and/or $E$. The value of the deviation is difficult to be established by a simple method.

The existence of the mirrors blocking and shading by the neighbouring heliostats does not introduce effects upon the angles $\theta$ and $\varphi$. But, taking into account these phenomena does not allow a simple redefinition of $l_{\mathrm{IH}}, l_{\mathrm{IV}}$ and $e$. We expect the concentration degree in the points $\mathrm{B}, \mathrm{D}, \mathrm{F}, \mathrm{G}, \mathrm{H}$, placed towards the middle of the total image not to be sensibly influenced.

The other simplifications used by us for HFT modelling (neglecting the redirected beam absorption in the atmosphere, the existence of certain inactive mirrors) seem to have mainly secondary effects and they haven't been systematically discussed by any of the authors quoted in our references.

The remarks made in this section have only an orientative character. The estimations, especially those concerning the influence of the tracking errors and mirrors surface imperfections are based on assumptions which haven't received a definite experimental validation. For illustration, we remind that the experimental verification of HELIOS program, which takes into account most of the disturbing phenomena enumerated above, show however, an error range of 10 per cent [30]. This fact also justifies the diversity of the opinions concerning the importance of the mentioned phenomena in the process of solar radiation concentration. 


\section{Conclusions.}

The heliostats field and the tower represents the most expensive part of a solar power plant. That is why, its design requires a special attention, in order that the investment helps an achievement with best technical and economical performances. As related to this subject, in the present paper we defined a given HFT from two points of view : (i) as related to its predefined structure, introducing a set of constructive and astronomical parameters and (ii) as related to its performances as a whole and those of the component elements, introducing a set of functional parameters.

In order to create an image as complete as possible, the interdependence between the two sets of parame- ters was separately studied for each parameter. The great number of parameters required to introduce a standard model on whose structure were made the simulations. That is why, our conclusions are directed in a certain measure by the standard model values, and their extrapolation especially in case of the fields of very great dimensions should be made carefully.

\section{Acknowledgments.}

I wish to thank Mr. R. Vasiloiu and Mr. D. Ciocionică from ICPE Bucharest for the computer program assistance. I also wish to thank the referee for his suggestions and remarks.

\section{References}

[1] Blake, F. A., Tracey, T. R., Walton, J. D., Bomar, S., Solar Energy 18 (1976) 513.

[2] Hildebrandt, A. F., HaAs, G. M., Jenkins, W. R., Colaco, J. P., Trans. Am. Geophys. Union 53 (1972) 684.

[3] MC FeE, R. H., Appl. Opt. 14 (1975) 1493.

[4] Aparissi, P. P., Malevsky, I. A., TarniJevsky, B. V., Goucev, V. K., KarpenKo, A. M., Revue Phys. Appl. 15 (1980) 357.

[5] Igel, E. A., Hughes, R. L., Solar Energy 22 (1979) 283.

[6] LIPPS, F. W., Solar Energy 18 (1976) 555.

[7] Sobin, A., WaGner, W., EASton, C. R., Solar Energy, 18 (1976) 21.

[8] Le Phat Vinh, A. and M., Rev. Gen. Thermique 192 (1977), 883.

[9] Malykoff, A. M., Aparissi, P. P., Revue Phys. Appl. 15 (1980) 377.

[10] Lipps, F. W., Walzel, M. D., Solar Energy 21 (1978) 113.

[11] Athavaley, K., Lipps, F., Vant-Hull, L., Solar Energy 22 (1979) 493.

[12] Fourakis, E., Severson, A. M., Solar Energy 19 (1977) 349.

[13] Moeller, C. E., Brumleve, T. D., Grosskreutz, C., SEAmONS, L. O., Solar Energy 25 (1980) 291.

[14] Achaibou, A., Bourdeau, C., Revue Phys. Appl. 15 (1980) 383.
[15] AватUt, J. L., Achaibou, A., Solar Energy 21 (1978) 453.

[16] Riaz, M., GurR, T., Solar Energy 19 (1977) 185.

[17] Boese, F. K., Merkel, A., Stahl, D., Stehle, H., Solar Energy 26 (1981) 1.

[18] JARVINEN, P. O., Solar Energy 19 (1977) 139.

[19] Trombe, F., Applications thermiques de l'énergie solaire dans le domaine de la recherche et de l'industrie (CNRS, Paris), 1961, p. 87-126.

[20] Mori, Y., Hijikata, K., Himeno, N., Nakayama, W., Solar Energy 19 (1977) 595.

[21] IPB Technical Report 305/1978.

[22] ITTNER III, W. B., Solar Energy 24 (1980) 221.

[23] LIPPS, F. W., Solar Energy 24 (1980) 461.

[24] Walzel, M. D., LipPs, F. W., Vant-Hull, L. L., Solar Energy 19 (1977) 239.

[25] WeI, L. Y., Solar Energy 26 (1981) 559.

[26] BĂdescu, V., VasiloIv', R., St. Cerc. Fiz., 36 (1984) 387.

[27] Bădescu, V., Revue Phys. Appl. 17 (1982) 421.

[28] BădesCU, V., St. Cerc. Fiz. 33 (1981) 455.

[29] Wijeysundera, N. E., Solar Energy 20 (1978) 81.

[30] Gintz, J., Bartlett, D., Zenter, R., Revue Phys. Appl. 15 (1980) 363.

[31] Hughes, R. O., Solar Energy 24 (1980) 83. 